\title{
Hypoglycemic and hypolipidemic effects of dietary supplementation of plantago psyllium seeds on diabetic and/ or hyperlipidemic albino rats
}

\author{
Zakia M. Abd el-kader, Hanaa M .Abd el-fattah, Mai E. Abd el-kawi and Asmaa I. Ali. \\ Biochemistry and Nutrition Department- Women's College, Ain Shams University, Cairo, \\ Egypt.
}

\begin{abstract}
This study was designed to investigate the hypoglycemic and hypolipidemic effects of plantago psyllium seeds added to diet in two different doses $(0.5 \%$ and $1.0 \% \mathrm{w} / \mathrm{w})$ on strepotozotocin (STZ) induced diabetic rats and/or hyperlipidemic rats. Eighty male adult albino rats were used in this study, divided into 10 groups 8 rats each. $G$ (1): served as healthy control, G (2) was diabetic control. G (3) was hyperlipidemic control. G (4) was diabetic fed on high fat high cholesterol diet (HFHC). Gs: (5-6) diabetic rats fed on diet supplemented with plantago psyllium seeds $(0.5 \%$ \& $1 \% \mathrm{w} / \mathrm{w})$.Gs $(7-8)$ hyperlipidemic rats fed on HFHC diet supplemented with plantago psyllium seeds $(0.5 \%$ \& $1 \% \mathrm{w} / \mathrm{w})$.Gs $(9-10)$ diabetic rats fed on HFHC diet supplemented with psyllium seeds $(0.5 \%$ \& $1 \% \mathrm{wlw})$. After 6 weeks, rats were sacrificed under diethyl ether anesthesia. Serum and tissue samples were collected to perform the biochemical analysis. Serum glucose, insulin, insulin resistance (HOMA-IR) and beta cell function index (HOMA- $\beta$ ) were measured. Moreover, Serum lipids profile including total lipid (TL), phospholipids (PL),total cholesterol (TC), triacylglycerols (TAGs),low density lipoprotein cholesterol (LDL-C),very low density lipoprotein cholesterol (VLDL-C), as well as LDL-C/ HDL-C, TC/HDL-C, TAGs/HDL-C ratios and atherogenic index (AI) were measured. Also, antioxidant status was evaluated by measuring total antioxidants capacity (TAC) level, glutathione peroxidase (GPx), glucose 6 phosphate dehydrogenase (G6PD) activities, malondialdehyde (MDA) and oxidative stress index (OSI). The results were supported with microscopic examination of pancreatic tissues. The results of this study revealed that administration of plantago psyllium seeds improve glucose homeostasis by decreasing blood glucose, increasing $\beta$-cell function, increasing serum insulin level and insulin sensitivity in STZ-induced diabetic rats. Also, plantago psyllium seeds ameliorate insulin resistance in hyperlipidemic groups by decreasing insulin level. The result of this study approved that psyllium seeds improve dyslipidemia relevant to HFHC diet and diabetes mainly through decreasing TL, PL, TC, TAGs, LDL-C, VLDL-C and increasing HDL-C. Furthermore, plantago psyllium seeds showed an important cardio protective effect through decreasing AI, TC/HDL-C,TAGs/HDL-C and LDL-C/ HDL-C ratios as a risk for coronary heart disease (CHD).The study revealed that plantago psyllium seeds has a significant antioxidant activity and can be used to protect tissue from oxidative stress as indicated in microscopic examination of pancreatic tissue showed amelioration in the appearance of cells .The result showed a significant decrease in serum MDA and OSI and a significant increase in TAC level ,GPx and G6PDactivities .The protective effect of plantago psyllium seeds is due to their antioxidant potential which represented by their rich content of dietary fibers, flavonoids, poly unsaturated fatty acids, and some polyphenols that appears to protect against reactive oxygen species (ROS).
\end{abstract}

Key Words: STZ, Psyllium, hyperlipidemia, fiber, antioxidants, oxidative stress, lipids profile.

Corresponding author:asmaaasmaaasmaa27@gmail.com 


\section{Introduction:}

Diabetes mellitus (DM) is a group of metabolic diseases characterized by hyperglycemia resulting from defects in insulin secretion, insulin action, or both. The chronic hyperglycemia of diabetes is associated with long-term tissue and organ damage, as well as, dysfunction involving the eyes, kidneys, nerves, heart, and blood vessels (American Diabetes Association, 2014). The presence of chronic hyperglycemia in DM is accompanied by greater or lesser impairment in the metabolism of carbohydrates, lipids and proteins. Also, hyperlipidemia is the most common complication of diabetes mellitus and it predisposes them to premature atherosclerosis and macrovascular complications. Diabetes and hyperlipidemia are two major factors involved in the development of cardiovascular disease (CVD) (Uttra et al., 2011).

Plants are well known in traditional herbal medicine for their hypoglycemic activities, and available literature indicate that there are more than 800 plants species showing hypoglycemic activities (Rajagopal and Sasikala,2008).There has been increasing demand for the use of plant products with anti-diabetic activity due to low cost, easy availability and lesser side effects. Epidemiological studies support an inverse relationship between dietary fiber and symptoms associated with type 2 DM and CVD (Aune et al., 2013). Importantly, whole grains are more strongly associated with decreased CVD risk than fruit or vegetablederived fibers. Specifically, whole grain fibers are more effective than fruit or vegetable fibers at reducing blood lipids, and inflammation as well as improving blood glucose in addition of blood pressure regulation (Satija and Hu, 2012). Much of this research has focused on the water-soluble fiber, psyllium (Plantago ovata), which is contained in the mucilage of the outer layer of the seed known as the seed coat. The mucilage is often referred to as husk, or ground psyllium husk (Johnson et al., 2017).

Psyllium (PSY) is classified as a mucilaginous fiber due to its powerful ability to form a gel in water. Plantago psyllium is an important medicinal plant which has different compounds such as phenolic compounds (Caffeic acid derivatives), flavonoids, alkaloids, terpenoids, and vitamin C. Plantago plants have high levels of phenols and the powerful antioxidant quality of the plant may be due to its phenolic compounds. Phenolic compounds, especially flavonoids have antioxidant qualities, but they are not able to inhibit all mutations induced by mutagenic agents (Haddadian et al., 2014). Psyllium contains nutritional antioxidants, flavonoids, polyunsaturated fatty acids (PUFAs), including essential fatty acids (Omega-3 and Omega-6 fatty acids), sulfur containing amino acids, and metabolites with bioactivities, which make it a promising candidate for use in the nutraceutical industry. Several studies suggested that psyllium improved glycemic and lipid control in individuals with diabetes. Psyllium intake has been shown to improve glucose and insulin responses in animal and human studies (Singh, 2007). Psyllium have hypolipidemic effect as it lowers serum cholesterol level as a result of the binding of bile acids in the intestinal lumen and reduced risk of coronary heart disease (Van Rosendaal et al., 2004).

So, this study was designed to investigate the hypoglycemic and hypolipidemic effects of psyllium seeds on diabetic and hyperlipidemic rats.

\section{Materials and Methods:}




\section{1- Materials:}

Adult male albino rats (Sprague Dawely strain ) weighing $150 \pm 5 \mathrm{~g}$ were obtained from the National Research Centre,(Giza, Egypt).STZ (N-Methyl-nitroso carbamoyl- $\alpha-$ D glucosamine) with molecular formula $\mathrm{C}_{8} \mathrm{H}_{15} \mathrm{~N}_{3} \mathrm{O}_{7}$ and molecular weight 265.22, was obtained from El-Gomohria Company for Chemical and Drugs Cairo, Egypt. Cholesterol

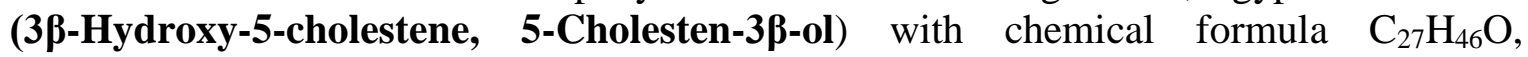
molecular weight 386.65, was obtained from El-Gomohria Company for Chemicals and Drugs, Cairo, Egypt .Standard balanced diet was used and prepared according to AIN 1993 and adjusted by Reeves et al. (1993). HFHC diet was prepared by modifying diet materials to enhance the elevation of serum total lipid and total cholesterol by adding $1 \%$ cholesterol and $4 \%$ beef tallow to the standard diet according to Pengzhan et al. (2003). Plantago psyllium seeds were obtained from local markets in Cairo. The seeds of plantago psyllium were oven dried at $60{ }^{\circ} \mathrm{C}$ for $1 / 2 \mathrm{hr}$ and were ground to make a powder using food processor grinder and added to diets as 0.5 or $1.0 \% \mathrm{w} / \mathrm{w}$.

\section{2- Methods:}

\subsection{1-Animal trial:}

Eighty adult male albino rats were randomly housed individually in metallic cages with constant controlled environments; temperature $25 \pm 3^{\circ} \mathrm{C}$ and $12 / 12 \mathrm{hr}$ light/dark cycle were held. The experiment was performed at the Animal House of National Cancer Institute. Experimental animals were fed with standard balanced diet and water ad libitum for 7 days as acclimation period. After the acclimation period, rats were divided into ten groups (8 rats/each), and hyperlipidemia was induced in six groups (G3,4,7,8,9 and 10) by feeding them the HFHC for 10 other days, while the remaining four groups (G1,2,5 and 6) continued feeding on the standard balanced diet. By the end of the ten days, the blood samples were collected from eye vein to confirm the hyperlipidemic condition through the determination of total cholesterol and triacylglycerols using Biodiagnostic kit according to Richmond (1973); and Fossati and prencipe(1982) respectively.

Then, four groups (G1,3,7 and 8) were injected interaperitoneally (i.p.) by saline solution and the remaining groups (G2,4,5,6,9and 10) were rendered diabetic by means of a single intraperitoneal (i.p.) injection of a freshly prepared STZ $(50 \mathrm{mg} / \mathrm{Kg} \mathrm{b.w}$.) dissolved in $0.1 \mathrm{M}$ sodium citrate buffer $\mathrm{pH} 4.5$ and injected within $15 \mathrm{~min}$ of preparation to rats Latha et al. (2014). As STZ is capable of inducing fatal hypoglycemia as a result of massive pancreatic insulin release; STZ-treated rats were allowed to drink 5\% glucose solution to overcome hypoglycemia after STZ injection. This was followed by monitoring random blood glucose level. The glucose conc. in blood taken from tail vein was determined by enzymatic colorimetric method according to Trinder (1969). Stable blood glucose level $\geq 200 \pm 5 \mathrm{mg} / \mathrm{dl}$ were considered to indicate diabetes onset .After the confirmation of diabetic and hyperlipidemic conditions of rats, the day on which rats were supplemented with plantago psyllium was considered as $1^{\text {st }}$ day of experiment.

The experimental groups were classified as follow: (G1) normal rats fed on standard balanced diet(-ve control) [NC], (G2) diabetic rats fed on standard balanced diet (Diabetic control) [DC], (G3) hyperlipidemic rats fed on HFHC diet (Hyperlipidemic control) [HC], (G4)diabetic hyperlipidemic rats fed on HFHC diet (Diabetic-hyperlipidemic control) [DHC], (G5)diabetic rats fed on diet supplemented with $0.5 \%$ Psy seeds[D+0.5\%Psy], (G6) diabetic rats fed on diet supplemented with $1 \%$ Psy seeds [D+1\% Psy], (G7) hyperlipidemic rats fed on HFHC diet supplemented with $0.5 \%$ Psy seeds [H+0.5\% Psy], (G8) hyperlipidemic rats fed on HFHC diet supplemented with1\% Psy seeds [H+1\%Psy], (G9) diabetic hyperlipidemic rats 
fed on HFHC diet supplemented with $0.5 \%$ Psy seeds [DH+0.5\% Psy], finally, (G10) diabetic hyperlipidemic rats fed on HFHC diet supplemented with $1.0 \%$ Psyllium seeds [DH+1\% Psy] (Ahmed et al ., 2010) .

Diet was introduced to the rats in special cups, water was introduced in a well-sealed bottles. All rats had free access to food and water all times for six weeks. Food intake was recorded daily and animals were weighed weekly to monitor the body weight changes and to calculate feed efficiency ratio (FER). At the end of the 6 weeks, and after $12 \mathrm{hrs}$ fasting, all rats were sacrificed under ether anesthesia and the blood samples were collected directly from hepatic portal vein Guo et al. (2002).

Blood samples were taken in two centrifuge tubes. The first tube was heparinized and immediately used for the determination of GPx and G6PD activities. The second tube was used for separation of serum left for 15 minutes at room temperature then centrifuged at 4000 rpm for 20 minutes, then a part of fresh serum samples were used immediately for the determination of glucose level and the others were separated and kept in plastic vials at $-20^{\circ} \mathrm{C}$ until analysis. Liver and Pancreas were separated immediately and washed by saline solution $(0.9 \% \mathrm{NaCl})$ then blotted on filter paper and weighed; part of pancreas were preserved in $10 \%$ neutral buffered formal saline for electron microscopic examination. Liver samples were stored at $-20^{\circ} \mathrm{C}$ until used for lipids profile determination.

\subsection{2- Biochemical analysis:}

Glucose in serum was performed by enzymatic colorimetric method using Vitro kits according to Trinder (1969). Serum insulin was determined using (Coat -A-Count) radioimmunoassay insulin kit (PITKIN-4) as described by Spin et al. (1998). The calculation of insulin resistance was performed according to the method of homeostasis model assessment of insulin resistance (HOMA) as described by Matthews et al. (1985). HOMA- $\beta$ scores were calculated using fasting serum insulin and fasting blood glucose concentrations at the end of the experimental period according to Wilson and Islam (2012). Determination of serum TL was performed by colorimetric method using Spin react kit according to Kaplan (1984). Serum PL were determined by colorimetric method using Spectrum kit according to Tietz (1990). Determination of serum TAGs was performed by enzymatic colorimetric method using Spectrum kit according to Fossati and prencipe (1982). Serum TC level was determined by enzymatic colorimetric method using Spectrum kit according to Richmond (1973).Serum HDL-C level was determined by enzymatic colorimetric method using spectrum kit according to Lopez-Virella et al. (1977). LDL-C level has been calculated indirectly by means of the Fridewald equation (Fridewald et al.,1972).Serum VLDL-C was calculated according to Fridewald et al. (1972). Atherogenic index was calculated according to Lecumberri et al. (2007). The risk ratio of LDL-C /HDL-C was calculated as described by Notarnicola et al. (2005). The risk ratio of TC/HDL-C was calculated according to Garg et al. (2014). The risk ratio of TAGs/HDL-C was calculated according to (Brehm et al., 2004). Determination of serum TAC was performed by colorimetric method using Biodiagnostic kit according to Koracevic et al. (2001). Serum MDA was determined according to Ohkawa et al. (1979) by thiobarbituric acid (TBA) method. The MDA/TAC ratio is proposed as oxidative stress index as reported by Suresh et al. (2010).Determination of GPx activity was carried according to Paglia and Valentina (1967).Determination of blood G6PD activity was performed by enzymatic colorimetric method using Biodiagnostic kit according to Dror et al. (1970). While, liver was homogenized with chloroform and methanol in a blender and then extracted by the method of Folch et al. (1957) for TC and TAGs level measurements. 


\subsection{3-Microscopic Examination:}

Pancreatic specimens from all animals were dissected immediately, and fixed in 10\% neutral-buffered formal saline for at least $72 \mathrm{hrs}$. All pancreatic specimens were washed in tap water for half an hr and then dehydrated in ascending grades of alcohol, cleared in xylene and embedded in paraffin. Serial sections of $6 \mu \mathrm{m}$ thick were cut and stained with haematoxylin and eosin for investigation according to Drury and Wallington (1980).

\subsection{Statistical Analysis:}

Data were statistically analyzed by Statistical Package for Social Science (SPSS) version 16.0 statistical packages. Values were presented as mean \pm SD. Statistical differences between groups were performed using one way ANOVA, the mean difference was significant at the level $(\mathrm{P} \leq 0.05)$ level according to Levesque (2007).

\section{Results}

\section{1- Biological measurements:}

Data in table (1) of nutritional evaluation showed that +ve control groups showed marked elevation in food intake as compared to -ve control group. On the other hand, diabetic and hyperlipidemic groups fed on psyllium seeds supplemented diets showed marked reduction $(\mathrm{P} \leq 0.05)$ in food intake as compared to +ve control groups. Generally, the reduction in food intake was dose dependent. While, diabetic hyperlipidemic groups fed on psyllium seeds supplemented diet showed significant increase $(\mathrm{P}<0.05)$ in food intake as compared to its corresponding control group. Concerning the results of body weight change, there was a significant reduction $(\mathrm{p} \leq 0.05)$ in body weight in diabetic control rats and diabetichyperlipidemic rats as compared to healthy group. While, there were remarkable increases $(\mathrm{p} \leq 0.05)$ in body weight in hyperlipidemic group as compared to healthy group.

From the present results, supplementation with Plantago psyllium seeds with the tested doses caused a significant increase $(p \leq 0.05)$ in body weight in diabetic groups when compared to their corresponding +ve control. While, the rate of weight gain in hyperlipidemic and diabetic hyperlipidemic groups supplemented with plantago psyllium seeds were lower than that in their corresponding positive control. With respect to the calculated FER which affected for far by the changes in body weight and food intake, data showed that there was a significant reduction $(\mathrm{p}<0.05)$ in FER in +ve control groups except hyperlipidemic group as compared to -ve control group . Our findings also demonstrated that dietary supplementation with $(0.5 \%$ and $1 \%)$ plantago psyllium seeds significantly improved $(\mathrm{p}<0.05)$ FER in diabetic treated groups. while, there were non significant change in FER in hyperlipidemic and diabetic hyperlipidemic treated groups as compared to their corresponding control.

Furthermore, data in table $(\mathbf{1})$ showed that there was a significant increase $(p<0.05)$ in relative liver weight in +ve control groups when compared to -ve control. The present results showed that addition of plantago psyllium seeds with two different doses caused a significant reduction $(\mathrm{p}<0.05)$ in relative liver weight in all treated groups. Considering the relative weight of pancreas, it's clear that there were a significant decrease $(p<0.05)$ in relative pancreatic weight in diabetic and diabetic hyperlipidemic groups as compared to healthy group. However, there was no significant difference in relative pancreatic weight between hyperlipidemic and healthy groups. The results demonstrated that there were a significant improvement $(\mathrm{p}<0.05)$ in relative pancreatic weight in diabetic and diabetic hyperlipidemic rats after dietary supplementation with two tested doses of plantago psyllium seeds. 
Table (1): Food intake, body weight change, feed efficiency ratio and relative organs weight (liver and pancreas) in experimental groups (mean \pm SD)

\begin{tabular}{|c|c|c|c|c|c|c|}
\hline Group no & Groups & Food intake & $\begin{array}{l}\text { Body weight } \\
\text { change } \\
(\mathrm{g})\end{array}$ & $\begin{array}{c}\text { Feed } \\
\text { efficiency } \\
\text { ratio } \\
\text { (FER) }\end{array}$ & $\begin{array}{c}\text { Relative } \\
\text { liver } \\
\text { weight } \\
(\mathrm{g} \%)\end{array}$ & $\begin{array}{c}\text { Relative } \\
\text { pancreatic } \\
\text { weight }(\mathrm{g} \%)\end{array}$ \\
\hline G1 & $\mathrm{NC}$ & $362.14 \pm 3.07^{\mathrm{a}}$ & $90.71 \pm 8.30^{\mathrm{a}}$ & $0.24 \pm 0.02^{\mathrm{a}}$ & $2.72 \pm 0.02^{f}$ & $2.34 \pm 0.05^{\mathrm{a}}$ \\
\hline G2 & $\mathrm{DC}$ & $412.28 \pm 6.29^{b}$ & $-43.14 \pm 4.25^{\mathrm{cd}}$ & $-0.10 \pm 0.01^{\mathrm{e}}$ & $3.92 \pm 0.02^{b}$ & $1.42 \pm 0.07^{\mathrm{e}}$ \\
\hline G3 & $\mathrm{HC}$ & $402.71 \pm 5.02^{\mathrm{c}}$ & $98 \pm 4.60^{\mathbf{c}}$ & $0.24 \pm 0.01^{\mathrm{a}}$ & $4.28 \pm 0.10^{\mathrm{a}}$ & $2.30 \pm 0.00^{\mathrm{a}}$ \\
\hline G4 & DHC & $367.42 \pm 4.79^{\mathrm{d}}$ & $48.42 \pm 2.07^{\mathrm{c}}$ & $0.13 \pm 0.01^{\mathrm{c}}$ & $4.28 \pm 0.07^{\mathrm{a}}$ & $1.67 \pm 0.04^{\mathrm{d}}$ \\
\hline G5 & $\mathrm{D}+0.5 \%$ psy & $382.57 \pm 1.98^{\mathrm{ef}}$ & $43.14 \pm 4.45^{\mathrm{cd}}$ & $0.11 \pm 0.01^{\mathrm{d}}$ & $3.0 \pm 0.07^{\mathrm{e}}$ & $2.00 \pm 0.05^{b c}$ \\
\hline G6 & $\mathrm{D}+1 \% \mathrm{psy}$ & $379.00 \pm 3.16^{\mathrm{e}}$ & $60.28 \pm 5.05^{b}$ & $0.15 \pm 0.01^{b}$ & $2.7 \pm 0.01^{f}$ & $2.28 \pm 0.08^{\mathrm{a}}$ \\
\hline G7 & $\mathrm{H}+0.5 \%$ psy & $384.71 \pm 3.14^{\mathrm{f}}$ & $31.71 \pm 3.86^{\mathrm{e}}$ & $0.08 \pm 0.00^{\mathrm{g}}$ & $3.44 \pm 0.08^{\mathrm{d}}$ & $2.04 \pm 0.53^{b}$ \\
\hline G8 & $\mathrm{H}+1 \%$ psy & $379.28 \pm 1.79^{\mathrm{e}}$ & $21.85 \pm 1.46^{\mathrm{f}}$ & $0.06 \pm 0.00^{\mathrm{g}}$ & $3.0 \pm 0.01^{\mathrm{e}}$ & $2.12 \pm 0.16^{b}$ \\
\hline G9 & $\mathrm{DH}+0.5 \% \mathrm{psy}$ & $389.00 \pm 2.16^{\mathrm{g}}$ & $31.42 \pm 4.31^{\mathrm{e}}$ & $0.04 \pm 0.00^{\mathrm{h}}$ & $3.61 \pm 0.07^{\mathrm{c}}$ & $2.04 \pm 0.53^{b}$ \\
\hline G10 & $\mathrm{DH}+1 \%$ psy & $383.00 \pm 2.70^{\mathrm{e}}$ & $16.85 \pm 2.96^{\mathrm{g}}$ & $0.08 \pm 0.01^{\mathrm{f}}$ & $3.10 \pm 0.69^{\mathrm{d}}$ & $2.31 \pm 0.08^{\mathrm{a}}$ \\
\hline LSD & & 3.90 & 4.80 & 0.0 & 0.07 & 0.08 \\
\hline
\end{tabular}

$\mathrm{P} \leq 0.05$, there are no significant difference between means have the same letters in the same column

\subsection{Biochemical measurements:}

Mean values of the statistical analysis present in table (2) showed that there were a significant increase $(\mathrm{P} \leq 0.5)$ in serum glucose and a significant decrease in serum insulin $(\mathrm{P} \leq$ 0.5) levels in diabetic and diabetic hyperlipidemic control groups comparing to normal control .While, there were a significant increase $(\mathrm{P} \leq 0.5)$ in serum insulin level in hyperlipidemic control rats with non-significant change in glucose level indicating increase risk of insulin resistance. Supplementation with psyllium seeds $(0.5 \& 1.0 \%)$ improved serum glucose and insulin levels in STZ-diabetic rats in a dose dependent manner .Moreover, hyperlipidemic rats that fed on psyllium supplemented diets showed marked reduction $(\mathrm{P}<0.05)$ in serum insulin level when compared with hyperlipidemic control group which indicating that psyllium seeds increase insulin sensitivity and decrease insulin resistance, the improvement in insulin level was dose dependent. There were a significant improvement in insulin resistance and sensitivity which illustrated by a significant decrease $(\mathrm{P} \leq 0.5)$ in homeostasis model assessment of insulin resistance (HOMA-IR) and significant elevation in pancreatic $\beta$ cell function (HOMA- $\beta$ ) score in diabetic treated groups. 
Table (2): Serum glucose, insulin levels and insulin resistance and $\beta$ - cell function indices in experimental groups (mean \pm SD)

\begin{tabular}{|c|c|c|c|c|c|}
\hline $\begin{array}{l}\text { Group } \\
\text { No. }\end{array}$ & $\begin{array}{l}\text { Parameters Group } \\
\text { Groups }\end{array}$ & $\begin{array}{l}\text { Glucose } \\
\text { (mg/dl) }\end{array}$ & $\begin{array}{l}\text { Insulin } \\
(\mu \mathrm{IU} / \mathrm{ml})\end{array}$ & $\begin{array}{c}\text { Insulin } \\
\text { resistance } \\
\text { (HOMA-IR) }\end{array}$ & $\begin{array}{c}\boldsymbol{\beta} \text {-cell function } \\
\text { index } \\
(\text { HOMA- } \beta)\end{array}$ \\
\hline G1 & $\mathrm{NC}$ & $152 \pm 1.63^{\mathrm{g}}$ & $10.14 \pm 0.14^{\mathrm{d}}$ & $3.8 \pm 0.05^{\mathrm{g}}$ & $41 \pm 1.13^{\mathrm{d}}$ \\
\hline $\mathrm{G} 2$ & $\mathrm{DC}$ & $391.85 \pm 1.95^{b}$ & $5.64 \pm 0.26^{1}$ & $5.4 \pm 0.27^{\mathrm{d}}$ & $6.1 \pm 0.29^{h}$ \\
\hline G3 & $\mathrm{HC}$ & $154.14 \pm 1.86^{\mathrm{g}}$ & $19.37 \pm 0.25^{\mathrm{a}}$ & $7.3 \pm 0.12^{\mathrm{a}}$ & $76.54 \pm 1.83^{\mathrm{a}}$ \\
\hline G4 & $\mathrm{DHC}$ & $434 \pm 2.0^{\mathrm{a}}$ & $6.58 \pm 0.19^{h}$ & $7.0 \pm 0.21^{\mathrm{b}}$ & $6.38 \pm 0.19^{h}$ \\
\hline G5 & $\mathrm{D}+0.5 \% \mathrm{psy}$ & $285.42 \pm 2.76^{\mathrm{d}}$ & $7.3 \pm 0.22^{g}$ & $5.12 \pm 0.09^{e}$ & $11.88 \pm 0.48^{\mathrm{g}}$ \\
\hline G6 & $\mathrm{D}+1.0 \% \mathrm{psy}$ & $184.14 \pm 4.67^{\mathrm{f}}$ & $9.5 \pm 0.17^{\mathrm{e}}$ & $4.28 \pm 0.12^{\mathrm{f}}$ & $28.38 \pm 1.0^{\mathrm{e}}$ \\
\hline G7 & $\mathrm{H}+0.5 \% \mathrm{psy}$ & $153.57 \pm 1.13^{\mathrm{g}}$ & $13.8 \pm 0.20^{\mathrm{b}}$ & $5.42 \pm 0.09^{\mathrm{d}}$ & $55.0 \pm 1.33^{\mathrm{b}}$ \\
\hline G8 & $\mathrm{H}+1.0 \%$ psy & $151.71 \pm 1.97^{\mathrm{g}}$ & $10.97 \pm 0.19^{c}$ & $4.1 \pm 0.08^{f}$ & $44.54 \pm 1.44^{\mathrm{c}}$ \\
\hline G9 & $\mathrm{DH}+0.5 \%$ psy & $317.85 \pm 4.22^{\mathrm{c}}$ & $8.0 \pm 0.08^{f}$ & $6.25 \pm 0.34^{\mathrm{c}}$ & $11.42 \pm 0.18^{\mathrm{g}}$ \\
\hline G10 & $\mathrm{DH}+1.0 \% \mathrm{psy}$ & $228.57 \pm 2.63^{\mathrm{e}}$ & $9.1 \pm 0.11^{\mathrm{e}}$ & $5.1 \pm 0.10^{\mathrm{e}}$ & $19.79 \pm 0.44^{\mathrm{f}}$ \\
\hline \multicolumn{2}{|c|}{$\mathrm{LSD}(\mathrm{P} \leq 0.05)$} & 2.89 & 0.65 & 0.19 & 1.07 \\
\hline
\end{tabular}

$\mathrm{P} \leq 0.05$, there are no significant difference between means have the same letters in the column

Results represented in table (3and 4) showed serum lipids profile and concluded that STZ-induced diabetes and HFHC diet caused marked elevation $(\mathrm{p}<0.05)$ in serum TL, PL , TC, TAGs , LDL-C, and VLDL-C levels and significant reduction $(\mathrm{p}<0.05)$ in serum HDL-C level as compared to healthy rats. The statistical analysis also indicated that supplementation of rat's diets with psyllium seeds by levels $(0.5 \& 1.0 \%)$ caused significant decrease $(p<0.05)$ in serum TL, PL, TC, TAGs, LDL-C, and VLDL-C levels and significant increase $(\mathrm{p}<0.05)$ in serum HDL-C level as compared to +ve control groups. The hypolipidemic effect of psyllium seeds was dose dependent.

Table (3): Serum total lipids (TL) ,phospholipids (PL) and triacylglycerols (TAGs) levels in experimental groups (mean $\pm \mathrm{SD})$.

\begin{tabular}{|l|l|c|c|c|}
\hline Group No. & Parameters & $\begin{array}{c}\text { TL } \\
(\mathbf{m g} / \mathbf{d l})\end{array}$ & $\begin{array}{c}\text { PL } \\
(\mathbf{m g} / \mathbf{d l})\end{array}$ & $\begin{array}{c}\text { TAGs } \\
(\mathbf{m g} / \mathbf{d l})\end{array}$ \\
\hline G1 & NC & $813.00 \pm 6.65^{\mathrm{I}}$ & $207.71 \pm 3.59^{\mathrm{j}}$ & $136.00 \pm 4.04^{\mathrm{h}}$ \\
\hline G2 & DC & $1113.14 \pm 6.74^{\mathrm{f}}$ & $385.57 \pm 3.20^{\mathrm{g}}$ & $203.28 \pm 3.77^{\mathrm{d}}$ \\
\hline G3 & HC & $21956.57 \pm 4.39^{\mathrm{c}}$ & $594.57 \pm 3.45^{\mathrm{d}}$ & $254.85 \pm 4.56^{\mathrm{b}}$ \\
\hline G4 & DHC & $994.57 \pm 3.73^{\mathrm{g}}$ & $356.71 \pm 5.46^{\mathrm{h}}$ & $165.42 \pm 3.73^{\mathrm{f}}$ \\
\hline G5 & D+0.5\%psy & $856.28 \pm 4.23^{\mathrm{h}}$ & $294.28 \pm 3.59^{\mathrm{I}}$ & $144.14 \pm 3.41^{\mathrm{g}}$ \\
\hline G6 & D+1.0\% psy & $1566.28 \pm 5.28^{\mathrm{d}}$ & $548.57 \pm 3.99^{\mathrm{e}}$ & $200.42 \pm 6.13^{\mathrm{d}}$ \\
\hline G7 & H+0.5\% psy & $1365.57 \pm 4.23^{\mathrm{e}}$ & $486.71 \pm 5.31^{\mathrm{f}}$ & $183.85 \pm 2.79^{\mathrm{e}}$ \\
\hline G8 & H+1.0\% psy & $1811.28 \pm 6.96^{\mathrm{b}}$ & $799.71 \pm 3.63^{\mathrm{b}}$ & $239.42 \pm 3.30^{\mathrm{c}}$ \\
\hline G9 & DH+0.5\% psy & $1653.14 \pm 8.57^{\mathrm{c}}$ & $737.57 \pm 5.15^{\mathrm{c}}$ & $199.85 \pm 3.48^{\mathrm{d}}$ \\
\hline G10 & DH+1.0\% psy & 6.15 & 4.52 & 4.2 \\
\hline LSD $(\mathrm{P} \leq 0.05)$ & &
\end{tabular}

$\mathrm{P} \leq 0.05$, there are no significant difference between means have the same letters in the same column 
Table (4): Serum total cholesterol (TC), low density lipoprotein cholesterol (LDL-C), very low density lipoprotein cholesterol (VLDL-C) and high density lipoproteins cholesterol (HDL-C) levels in experimental groups (mean \pm SD).

\begin{tabular}{|l|l|c|c|c|c|}
\hline $\begin{array}{l}\text { Group } \\
\text { No. }\end{array}$ & \multicolumn{1}{|c|}{ parameters } & $\begin{array}{c}\text { TC } \\
(\mathbf{m g} / \mathbf{d l})\end{array}$ & $\begin{array}{c}\text { LDL-C } \\
(\mathbf{m g} / \mathbf{d})\end{array}$ & $\begin{array}{c}\text { VLDL-C } \\
(\mathbf{m g} / \mathbf{d l})\end{array}$ & $\begin{array}{c}\text { HDL-C } \\
(\mathbf{m g} / \mathbf{d l})\end{array}$ \\
\hline G1 & NC & $120.28 \pm 3.45^{\mathrm{l}}$ & $49.22 \pm 4.36^{\mathrm{h}}$ & $27.20 \pm 0.80^{\mathrm{g}}$ & $43.88 \pm 3.25^{\mathrm{a}}$ \\
\hline G2 & DC & $164.28 \pm 3.68^{\mathrm{f}}$ & $103.60 \pm 5.11^{\mathrm{e}}$ & $37.11 \pm 0.83^{\mathrm{d}}$ & $23.00 \pm 2.16^{\mathrm{e}}$ \\
\hline G3 & HC & $256.28 \pm 5.12^{\mathrm{c}}$ & $188.82 \pm 5.63^{\mathrm{c}}$ & $47.28 \pm 0.74^{\mathrm{b}}$ & $20.17 \pm 1.91^{\mathrm{f}}$ \\
\hline G4 & DHC & $328.00 \pm 4.93^{\mathrm{a}}$ & $253.30 \pm 5.01^{\mathrm{a}}$ & $58.20 \pm 0.68^{\mathrm{a}}$ & $16.50 \pm 1.12^{\mathrm{g}}$ \\
\hline G5 & D+0.5 \%psy & $144.00 \pm 3.55^{\mathrm{g}}$ & $80.77 \pm 3.67^{\mathrm{f}}$ & $33.08 \pm 0.71^{\mathrm{e}}$ & $30.14 \pm 2.41^{\mathrm{c}}$ \\
\hline G6 & D+1.0\% psy & $127.14 \pm 4.59^{\mathrm{h}}$ & $57.02 \pm 3.89^{\mathrm{g}}$ & $28.82 \pm 0.68^{\mathrm{f}}$ & $41.28 \pm 4.23^{\mathrm{a}}$ \\
\hline G7 & H+0.5\% psy & $206.57 \pm 4.31^{\mathrm{d}}$ & $138.07 \pm 7.02^{\mathrm{d}}$ & $39.66 \pm 0.77^{\mathrm{c}}$ & $28.14 \pm 1.86^{\mathrm{c}}$ \\
\hline G8 & H+1.0\% psy & $185.14 \pm 3.48^{\mathrm{e}}$ & $107.15 \pm 2.74^{\mathrm{e}}$ & $36.77 \pm 0.55^{\mathrm{d}}$ & $35.00 \pm 3.05^{\mathrm{b}}$ \\
\hline G9 & DH+0.5\% psy & $269.14 \pm 3.62^{\mathrm{b}}$ & $194.25 \pm 5.23^{\mathrm{b}}$ & $47.88 \pm 0.66^{\mathrm{b}}$ & $27.00 \pm 2.16^{\mathrm{cd}}$ \\
\hline G10 & DH+1.0\% psy & $208.57 \pm 4.85^{\mathrm{d}}$ & $135.40 \pm 5.82^{\mathrm{d}}$ & $39.97 \pm 0.69^{\mathrm{c}}$ & $33.20 \pm 2.00^{\mathrm{b}}$ \\
\hline LSD (P $\leq 0.05)$ & 4.49 & 5.30 & 0.77 & 2.73 \\
\hline
\end{tabular}

$\mathrm{P} \leq 0.05$ there are no significant difference between means have the same letters in the same column

Moreover, the data presented in table (5) showed heart disease risks. It was clear from results that there were a significant increase in several atherogenic indices TC/HDL-C, TAGs/HDL-C, LDL-C/HDL-C ratios and AI in all +ve control groups as increase risk of coronary heart disease (CHD).Then after supplementation of psyllium (two doses) there were a significant reduction $(\mathrm{P}<0.05)$ in TC/HDL-C, TAGs/HDL-C, LDL-C/HDL-C and AI which indicated that psyllium fiber may provide cardio-protective benefits.

Table (5 ): Serum TC/HDL-C , TAGs/HDL-C , LDL-C/ HDL-C ratios and AI in experimental groups $($ mean $\pm \mathrm{SD})$.

\begin{tabular}{|c|c|c|c|c|c|}
\hline $\begin{array}{l}\text { Group } \\
\text { NO. }\end{array}$ & Groups & $\begin{array}{c}\text { TC / HDL-C } \\
\text { Ratio }\end{array}$ & $\begin{array}{c}\text { TAGs/HDL-C } \\
\text { ratio }\end{array}$ & $\begin{array}{c}\text { LDL/HDL-C } \\
\text { ratio }\end{array}$ & AI \\
\hline G1 & $\mathrm{NC}$ & $2.75 \pm 0.21^{\mathrm{g}}$ & $3.11 \pm 0.23^{\mathrm{f}}$ & $1.13 \pm 0.16^{\mathrm{g}}$ & $1.75 \pm 0.21^{I}$ \\
\hline $\mathrm{G} 2$ & $\mathrm{DC}$ & $7.20 \pm 0.80^{\mathrm{d}}$ & $8.91 \pm 0.91^{\mathrm{c}}$ & $4.55 \pm 0.60^{\mathrm{d}}$ & $6.20 \pm 0.80^{\mathrm{d}}$ \\
\hline G3 & $\mathrm{HC}$ & $12.80 \pm 1.30^{b}$ & $12.73 \pm 1.24^{\mathrm{b}}$ & $9.44 \pm 1.07^{b}$ & $11.80 \pm 1.30^{\mathrm{b}}$ \\
\hline G4 & DHC & $19.95 \pm 1.28^{\mathrm{a}}$ & $17.70 \pm 1.14^{\mathrm{a}}$ & $15.41 \pm 1.07^{\mathrm{a}}$ & $18.95 \pm 1.28^{\mathrm{a}}$ \\
\hline G5 & $\mathrm{D}+0.5 \%$ psy & $4.80 \pm 0.36^{\mathrm{f}}$ & $5.52 \pm 0.54^{\mathrm{e}}$ & $2.69 \pm 0.26^{\mathrm{f}}$ & $3.80 \pm 0.36^{\mathrm{g}}$ \\
\hline G8 & $\mathrm{D}+1.0 \% \mathrm{psy}$ & $3.10 \pm 0.26^{\mathrm{g}}$ & $3.51 \pm 0.31^{f}$ & $1.39 \pm 0.20^{\mathrm{g}}$ & $2.01 \pm 0.26^{\mathrm{h}}$ \\
\hline G6 & $\mathrm{H}+0.5 \%$ psy & $7.36 \pm 0.51^{d}$ & $7.14 \pm 0.50^{\mathrm{d}}$ & $4.92 \pm 0.48^{\mathrm{d}}$ & $6.36 \pm 0.51^{d}$ \\
\hline G9 & $\mathrm{H}+1.0 \%$ psy & $5.32 \pm 0.44^{\mathrm{f}}$ & $5.29 \pm 0.51^{\mathrm{e}}$ & $3.08 \pm 0.29^{f}$ & $4.32 \pm 0.44^{\mathrm{f}}$ \\
\hline G7 & $\mathrm{DH}+0.5 \%$ psy & $10.02 \pm 0.88^{\mathrm{c}}$ & $8.91 \pm 0.70^{\mathrm{c}}$ & $7.24 \pm 0.74^{\mathrm{c}}$ & $9.02 \pm 0.88^{\mathrm{c}}$ \\
\hline G10 & $\mathrm{DH}+1.0 \%$ psy & $6.30 \pm 0.42^{\mathrm{e}}$ & $6.04 \pm 0.42^{\mathrm{e}}$ & $4.09 \pm 0.35^{\mathrm{de}}$ & $5.30 \pm 0.42^{\mathrm{e}}$ \\
\hline \multicolumn{2}{|c|}{$\mathrm{LSD}(\mathrm{P} \leq 0.05)$} & 0.8 & 0.78 & 0.6 & 0.8 \\
\hline
\end{tabular}

$\mathrm{P} \leq 0.05$, there are no significant difference between means have the same letters in the same column

Furthermore, results in table (6 and 7) indicated that diabetes mellitus and hyperlipidemia increased lipid peroxidation that was manifested by marked elevation $(\mathrm{p}<0.05)$ in serum MDA level in +ve control groups as compared to -ve control groups. Moreover, there was a disturbance in antioxidants status that was showed in the significant reduction of total antioxidants capacity (TAC) level, GPx andG6PD activities as compared to 
-ve control group. Also, there was a significant elevation $(\mathrm{P}<0.05)$ in MDA/TAC ratio. On the other hand, supplementation of experimental diets with psyllium seeds $(0.5 \& 1.0 \%)$ improved lipid peroxidation and antioxidants status that was showed by marked reduction $(\mathrm{p}<0.05)$ in serum MDA level and MDA/TAC ratio and significant elevation $(\mathrm{P}<0.05)$ of serum TAC level and GPx and G6PD activities in all treated groups as compared to their corresponding +ve control groups. Generally, this improvement was dose dependent.

Table (6): Serum malondialdehyde (MDA) and total antioxidant capacity (TAC) levels and oxidative stress index in experimental groups (means \pm SD).

\begin{tabular}{|c|c|c|c|c|}
\hline Group NO. & $\begin{array}{l}\text { Parameters } \\
\text { Groups }\end{array}$ & $\begin{array}{c}\text { MDA } \\
(\mathbf{n m o l} / \mathbf{m l})\end{array}$ & $\begin{array}{c}\text { TAC } \\
(\mathrm{mM} / \mathrm{L})\end{array}$ & $\begin{array}{c}\text { MDA/TAC } \\
\text { (OSI) }\end{array}$ \\
\hline G1 & $\mathrm{NC}$ & $133.14 \pm 1.95^{1}$ & $1.02 \pm 0.01^{\mathrm{a}}$ & $130.14 \pm 1.57^{1}$ \\
\hline G2 & DC & $241.71 \pm 1.49^{d}$ & $0.41 \pm 0.01^{\mathrm{g}}$ & $581.42 \pm 4.72^{\mathrm{c}}$ \\
\hline G3 & $\mathrm{HC}$ & $253.40 \pm 3.0^{\mathrm{c}}$ & $0.51 \pm 0.01^{\mathrm{f}}$ & $498.28 \pm 5.8^{\mathrm{d}}$ \\
\hline G4 & DHC & $311.42 \pm 7.20^{\mathrm{a}}$ & $0.31 \pm 0.01^{\mathrm{h}}$ & $1004 \pm 4.6^{\mathrm{a}}$ \\
\hline G5 & $\mathrm{D}+0.5 \% \mathrm{psy}$ & $197.57 \pm 2.07^{\mathrm{e}}$ & $0.64 \pm 0.02^{\mathrm{e}}$ & $308.28 \pm 6.26^{\mathrm{e}}$ \\
\hline G6 & $\mathrm{D}+1.0 \% \mathrm{psy}$ & $164.00 \pm 2.30^{\mathrm{g}}$ & $0.87 \pm 0.01^{b}$ & $188.57 \pm 2.76^{\mathrm{g}}$ \\
\hline G7 & $\mathrm{H}+0.5 \%$ psy & $190.42 \pm 1.71^{f}$ & $0.71 \pm 0.01^{d}$ & $268.71 \pm 3.25^{\mathrm{f}}$ \\
\hline G8 & $\mathrm{H}+1.0 \%$ psy & $158.00 \pm 2.16^{\mathrm{h}}$ & $0.91 \pm 0.01^{b}$ & $174.28 \pm 3.14^{\mathrm{h}}$ \\
\hline G9 & $\mathrm{DH}+0.5 \% \mathrm{psy}$ & $282.85 \pm 3.53^{\mathrm{b}}$ & $0.51 \pm 0.01^{\mathrm{f}}$ & $559.42 \pm 1.81^{\mathrm{b}}$ \\
\hline G10 & $\mathrm{DH}+1.0 \% \mathrm{psy}$ & $243.14 \pm 2.04^{\mathrm{d}}$ & $0.79 \pm 0.01^{\mathrm{c}}$ & $306.85 \pm 3.48^{\mathrm{e}}$ \\
\hline $\operatorname{LSD}(\mathrm{P} \leq 0.05)$ & & 3.4 & 0.05 & 4.32 \\
\hline
\end{tabular}

$\mathrm{P} \leq 0.05$, there are no significant difference between means have the same letters in the same column

Table (7): Blood glutathione peroxidase (GPx) and Glucose 6 phosphate dehydrogenase (G6PD) activities in the experimental groups (mean \pm SD).

\begin{tabular}{|l|l|c|c|}
\hline Group No. & Parameters & $\begin{array}{c}\text { GPx activity } \\
(\mathbf{m U} / \mathbf{m l})\end{array}$ & $\begin{array}{c}\text { G6PD activity } \\
\text { (U/g Hb) }\end{array}$ \\
\hline G1 & NC & $470.42 \pm 1.71^{\mathrm{a}}$ & $14.18 \pm 0.56^{\mathrm{a}}$ \\
\hline G2 & DC & $362.14 \pm 1.57^{\mathrm{d}}$ & $8.04 \pm 0.26^{\mathrm{g}}$ \\
\hline G3 & HC & $361.57 \pm 4.11^{\mathrm{d}}$ & $10.07 \pm 0.42^{\mathrm{e}}$ \\
\hline G4 & DHC & $302.57 \pm 5.41^{\mathrm{f}}$ & $6.04 \pm 0.29^{\mathrm{h}}$ \\
\hline G5 & D+0.5\%psy & $400.57 \pm 5.09^{\mathrm{b}}$ & $9.40 \pm 0.22^{\mathrm{f}}$ \\
\hline G6 & D+1.0\% psy & $467.14 \pm 1.67^{\mathrm{a}}$ & $12.07 \pm 0.36^{\mathrm{c}}$ \\
\hline G7 & H+0.5\% psy & $400.71 \pm 1.79^{\mathrm{b}}$ & $11.48 \pm 0.32^{\mathrm{d}}$ \\
\hline G8 & H+1.0\% psy & $468.42 \pm 1.90^{\mathrm{a}}$ & $13.08 \pm 0.30^{\mathrm{b}}$ \\
\hline G9 & DH+0.5\% psy & $345.00 \pm 4.08^{\mathrm{e}}$ & $7.98 \pm 0.33^{\mathrm{g}}$ \\
\hline G10 & DH+1.0\% psy & $394.28 \pm 3.68^{\mathrm{c}}$ & $9.44 \pm 0.25^{\mathrm{f}}$ \\
\hline LSD $(\mathrm{P} \leq 0.05)$ & & 3.66 & 0.365 \\
\hline P $\leq 0.05, \mathrm{~m}$ & &
\end{tabular}

$\mathrm{P} \leq 0.05$, there are no significant difference between means have the same letters in the same column

The results presented in Table (8), showed the hepatic TC and hepatic TAGs levels. The results indicated that there were a significant elevation in TC and TAGs in liver tissue in all +ve control groups as compared to negative control .While psyllium supplementation resulted in a significant reduction in hepatic lipids in all treated groups as compared to their corresponding controls. 
Table (8): Total cholesterol (TC) and triacylglycerols (TAGs) levels in liver tissue of experimental groups (means $\pm \mathrm{SD}$ ).

\begin{tabular}{|l|l|c|c|}
\hline \multicolumn{1}{|c|}{ Group No. } & $\begin{array}{c}\text { Parameters } \\
\text { Groups }\end{array}$ & $\begin{array}{c}\text { TC } \\
(\mathbf{m g} / \mathbf{g} \text { tissue })\end{array}$ & $\begin{array}{c}\text { TAGs } \\
(\mathbf{m g} / \mathbf{g} \text { tissue })\end{array}$ \\
\hline G1 & NC & $2.9 \pm 0.17^{\mathrm{h}}$ & $3.1 \pm 0.18^{\mathrm{h}}$ \\
\hline G2 & DC & $4.6 \pm 0.30^{\mathrm{e}}$ & $4.45 \pm 0.17^{\mathrm{e}}$ \\
\hline G3 & HC & $6.4 \pm 0.21^{\mathrm{b}}$ & $5.4 \pm 0.34^{\mathrm{c}}$ \\
\hline G4 & DHC & $7.3 \pm 0.21^{\mathrm{a}}$ & $6.7 \pm 0.17^{\mathrm{a}}$ \\
\hline G5 & D+0.5\%psy & $3.9 \pm 0.17^{\mathrm{g}}$ & $3.9 \pm 0.13^{\mathrm{f}}$ \\
\hline G6 & D+1\% psy & $3.0 \pm 0.19^{\mathrm{h}}$ & $3.5 \pm 0.31^{\mathrm{g}}$ \\
\hline G7 & H+0.5\% psy & $5.2 \pm 0.23^{\mathrm{d}}$ & $4.68 \pm 0.24^{\mathrm{e}}$ \\
\hline G8 & H+1\% psy & $4.2 \pm 0.24^{\mathrm{f}}$ & $3.6 \pm 0.20^{\mathrm{g}}$ \\
\hline G9 & DH+0.5\%psy & $6.7 \pm 0.17^{\mathrm{c}}$ & $5.9 \pm 0.17^{\mathrm{b}}$ \\
\hline G10 & DH+1\%psy & $5.4 \pm 0.39^{\mathrm{d}}$ & $5.0 \pm 0.21^{\mathrm{d}}$ \\
\hline LSD & & 0.25 & 0.24 \\
\hline
\end{tabular}

$\mathrm{P} \leq 0.05$, there are no significant difference between means have the same letters in the same column

\section{3- Microscopic examination:}

It was clear from the microscopic examination of pancreas that in Fig (1), normal control group revealed no histopathological changes. It showed the normal structure of the tissue, the pancreatic endocrine island with normal cellular archtecture with their normal secretory activity, normal exocrine architecture with normal acina . Also, the blood vessel with normal thickness without any deposited material as well as the exocrine acini which appear normal. .On the other hand, Fig ( 2 ) showed section of pancreas from diabetic control rats characterized by reduced cellularity of pancreatic island which replaced by hyaline deposition. The blood vessels wall thickened due to the hyaline deposition. These findings explain the reduction in insulin secretion and hyperglycemia which occurs as a consequence of STZ induction.

Our results provided that, a section of the pancreatic tissue of hyperlipidemic rat showed vacuolation of the exocrine pancreatic tissue due to fatty acid deposition, on the other hand ,the pancreatic island without any damage noticed as illustrated in Fig (3) . a section of the pancreatic tissue of diabetic hyperlipidemic rats showed decreased cellularity of pancreatic island which replaced by hyaline deposition with vacuolation of the exocrine pancreatic tissue due to fatty acid deposition as illustrated in Fig (4).

Fig (5) showed pancreas from diabetic rats treated with $0.5 \%$ psyllium seeds that's characterized by the pancreatic islands which still destructed and minimal hyaline deposition and also the blood vessels retain its thickness with minimal hyaline deposition. While, Fig (6) a section of the pancreatic tissue of the diabetic rat with $1 \%$ psyllium seeds showing that the pancreatic island get improved and retain its cellularity, also the secretory activity of the cells get improved.

Fig (7) showed a section of the pancreatic tissue of hyperlipidemic rat with $0.5 \%$ psyllium seeds showing the pancreatic endocrine island with more normal cellularity and good secretory activity, on the other hand the exocrine pancreatic tissue noticed to be normal without the fatty deposits. While Fig (8) showed a section of the pancreatic tissue of the hyperlipidemic rat with $1 \%$ psyllium seeds showing that the pancreatic island get improved and retain its cellularity, also the secretory activity of the cells get improved.

Fig (9) showed a section of the pancreatic tissue of diabetic hyperlipidemic rat with $0.5 \%$ psyllium seeds indicate a normal pancreatic endocrine island but still with minimal cellularity and hyaline deposition on the blood vessel wall, on the other hand there is no appearance to the fatty deposition in the exocrine pancreatic tissue .On the other hand Fig 
(10) showed a section of the pancreatic tissue of the diabetic hyperlipidemic rat $1 \%$ psyllium seeds indicated that the pancreatic island get improved without fatty acids depositions in the exocrine pancreatic tissue, also in the left side showed that the blood vessels with normal thickness without hyaline deposition in its wall.

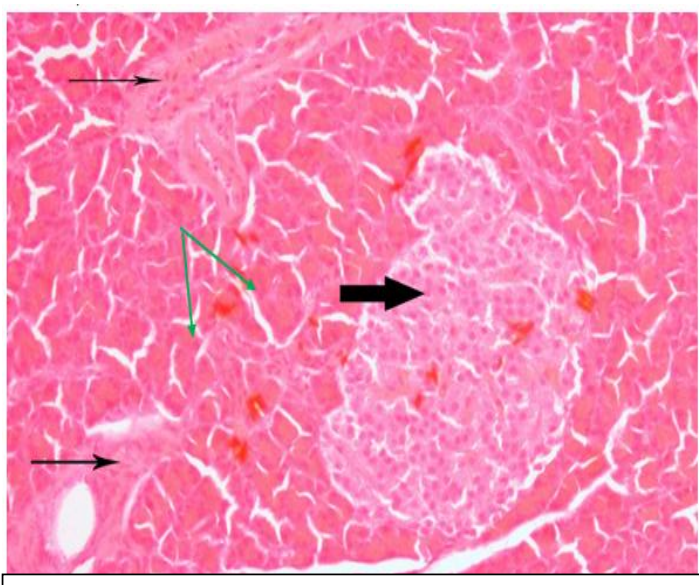

Figure (1) A photomicrograph of a section of pancreas in healthy rats showed normal histological structure of islet cells. (Hx\& E. X 200)

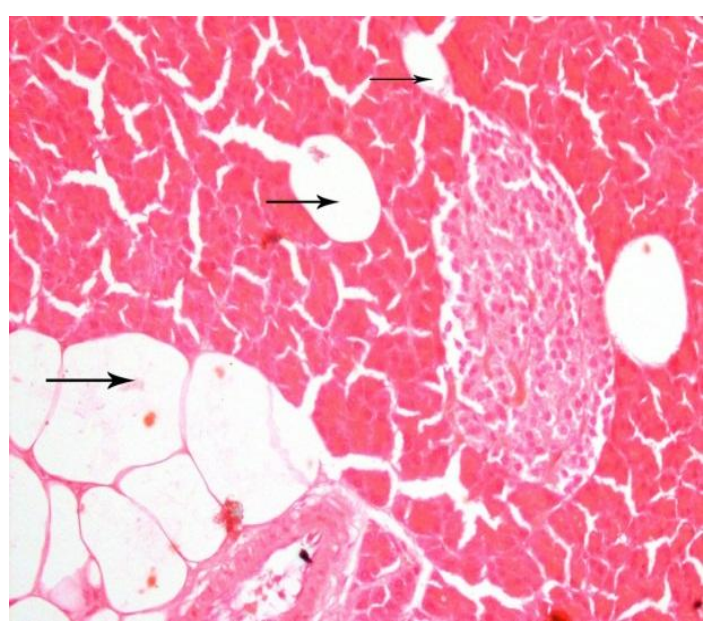

Figure (3) A photomicrograph of a section of pancreas in hyperlipidemic rats with vacuolation in the exocrine pancreatic tissue without any damage noticed (Hx\& E.X 200)
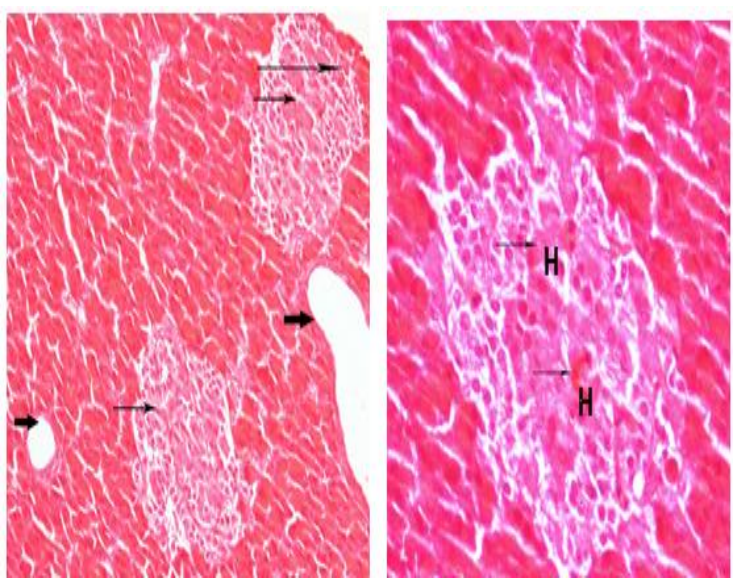

Figure (2) A photomicrograph of a section of the pancreatic tissue of a diabetic rat showing in the right side the pancreatic island with reduced cellularity which replaced by hyaline deposition $(\mathbf{H})$ also the blood vessels wall thickened due to the hyaline deposition (thick arrow), The left side showing the hyaline deposition in the pancreatic tissue (thin arrow)(Hx\& E. X 200, 400)

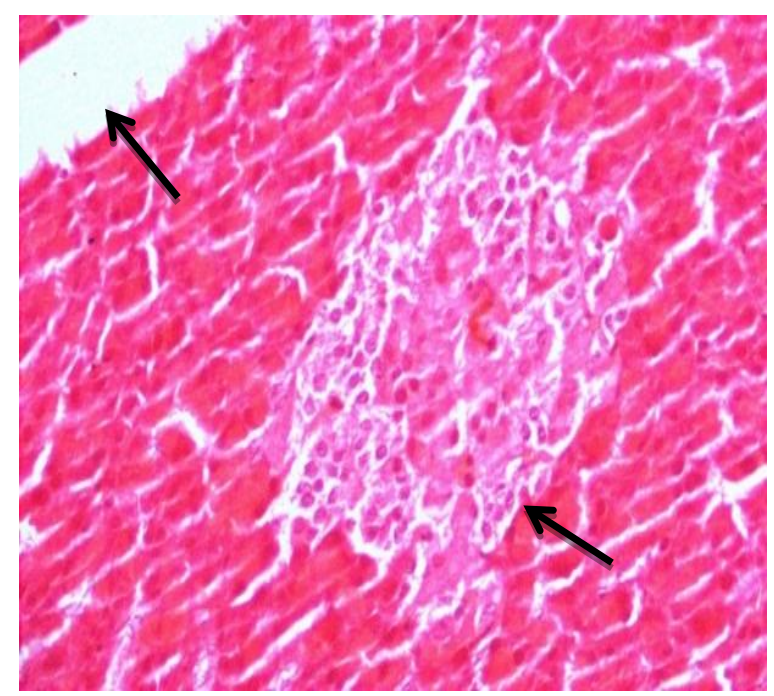

Figure (4) A photomicrograph of a section of pancreas in diabetic hyperlipidemic rats showed the pancreatic island with reduced cellularity, thickened blood vessels and vacuolation in the exocrine pancreatic tissue (Hx \& E. X 200 ). 


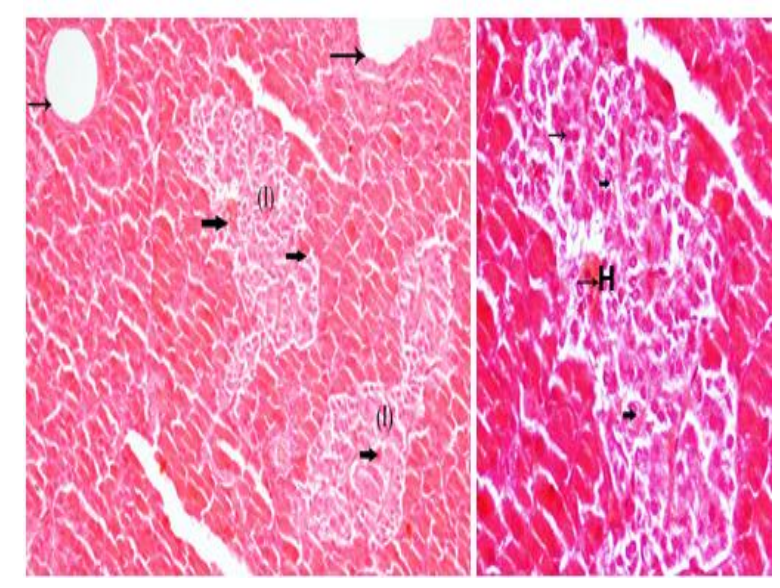

Figure (5) A photomicrograph of a section of pancreas of D+0.5\% \%sy seeds group which still destructed and minimal hyaline deposition $(\mathrm{H})$ and also the blood vessels retain its thickness $(\mathrm{Hx} \& \mathrm{E}$. X 200, 400)

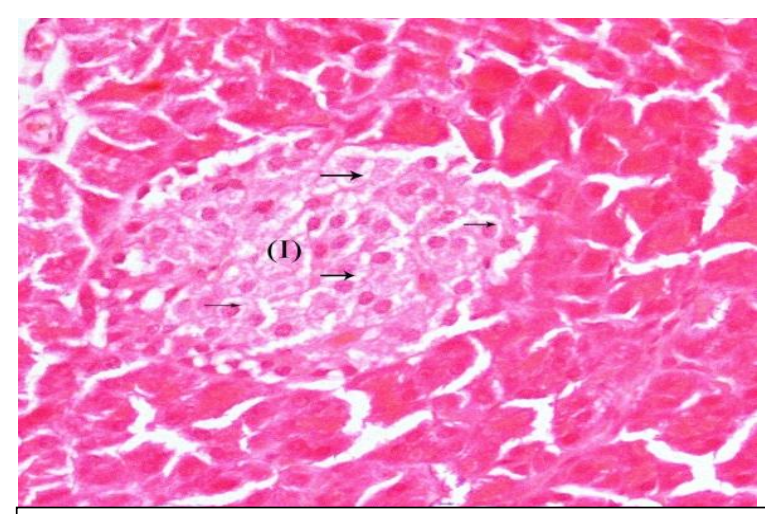

Figure (7) A photomicrograph of a section of the pancreatic tissue of $\mathrm{H}+0.5 \%$ Psy seeds group with normal cellularity without fatty acid deposition (Hx\& E. X 200 )

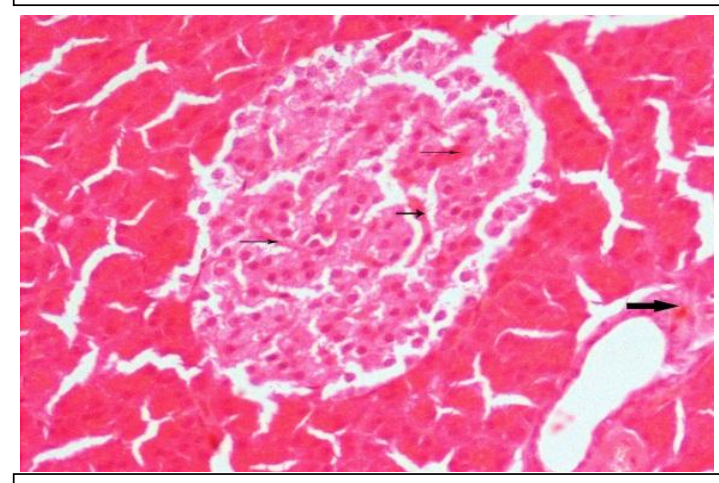

Figure (9) A photomicrograph of a section of the pancreatic tissue of $\mathrm{DH}+0.5 \%$ Psy seeds group showing normal pancreatic endocrine island but still with minimal cellularity (thin arrows) and hyaline deposition on the blood vessel wall (thick arrows) (Hx\& E. X 200 )

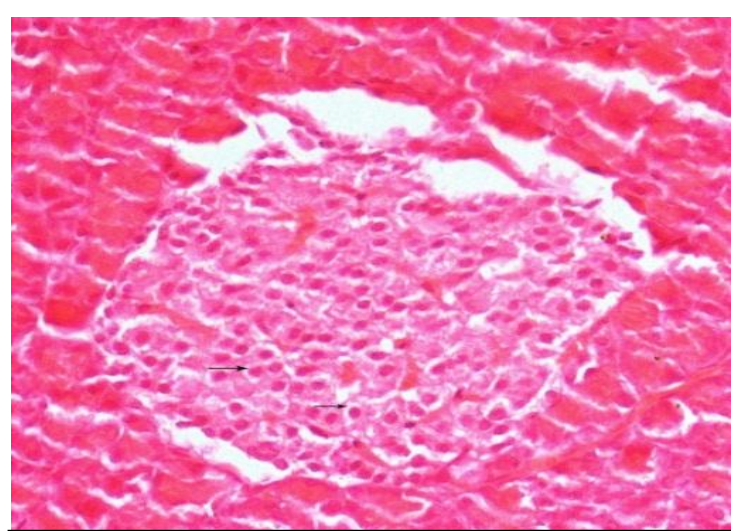

Figure (6) A photomicrograph of section of pancreatic tissue of the $\mathrm{D}+1.0 \%$ Psy seeds group showing that the pancreatic island get improved and retain its cellularity, also the secretory activity of the cells get improved (arrows). ( $\left.\mathrm{H}_{\mathrm{X}} \& \mathrm{E} . \mathrm{X} 200\right)$

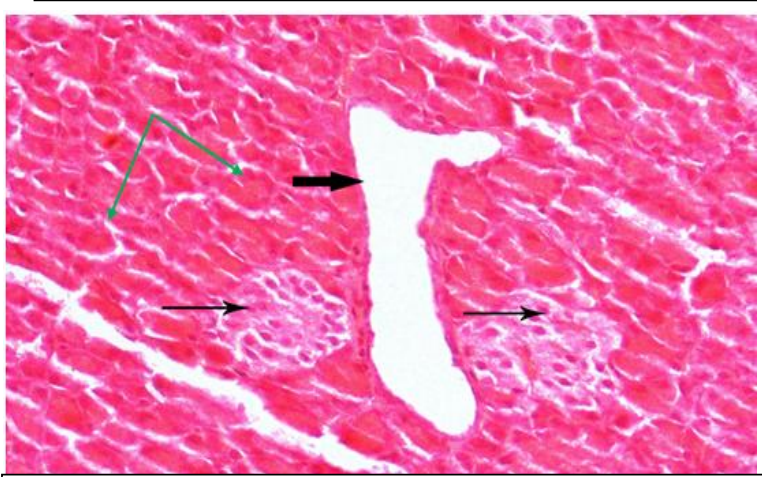

Figure (8) A photomicrograph of a section of the pancreatic tissue of $\mathrm{H}+1.0 \%$ Psy seeds group showing normal pancreatic island (thin arrows) with normal exocrine part as normal looking acini (green arrows) also blood vessels look like normal. $\left(\mathrm{H}_{\mathrm{X}} \& \mathrm{E} . \mathrm{X} 200\right)$

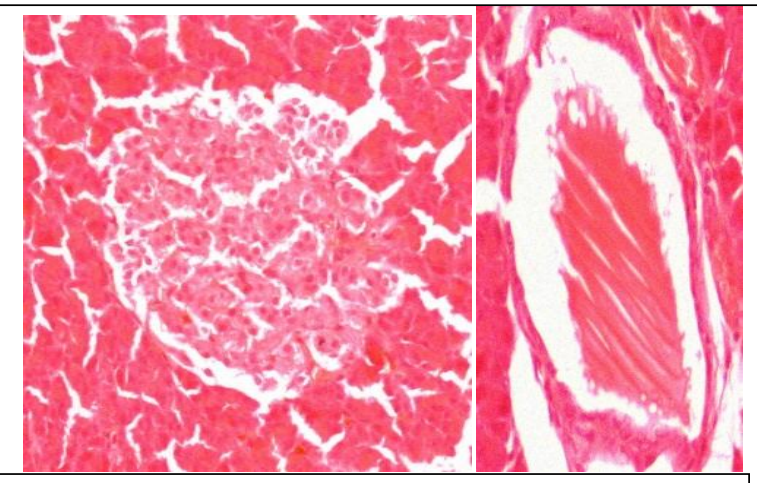

Figure (10) A photomicrograph of a section of the pancreatic tissue of the DH rat with $1 \%$ Psy seeds group showing in the right side that the pancreatic island get improved without fatty acids depositions in the exocrine pancreatic tissue ,also in the left side showing the blood vessels with normal thickness without hyaline deposition in its wall (Hx\& E. X 200, 400 ) 


\section{Discussion:}

\section{4- Discussion :}

The goal of the present study was designed to investigate the anti-diabetic and/ or the hypolipidemic effects of plantago psyllium seeds added to diet in two tested doses at $(0.5$ and $1 \% \mathrm{w} / \mathrm{w})$ in experimental groups which were fed on balanced diet or HFHC diet by measuring changes in the biological and biochemical parameters.

The results of the present study showed that injection with STZ produced hyperglycemia and hypoinsulinemia in rats .On the other hand, psyllium seeds reversed STZinduced change in glucose and insulin levels.STZ enter $\beta$ cells via a glucose transporter (GLUT2) using a variety of intracellular toxic mechanisms such as production of oxygen free radicals that causes degeneration of pancreatic $\beta$-cells leading to hypoinsulinemia and subsequent hyperglycemia (Bhateja and Singh, (2016); and Guang-Kai et al.,(2017).

Moreover, induction of hyperlipidemia caused insulin resistance which indicated by compensatory hyperinsulinaemia and marked increase in HOMA-IR. In agreement with our study Ribeiro et al. (2012) found that experimental model fed on a HFHC diet led to obesity and insulin resistance which reflected by hyperinsulinemia . Similarly, Haroun et al. (2011) investigated that both HFHC and high fructose diets intake caused insulin resistance with hyperglycemia, hyperinsulineamia, hyperlipidemia and hypertension in rats.

Also, in agreement with our results Karhunen et al. (2010) found that psyllium fiber enriched meals improve glucose and insulin level significantly than non- fiber enriched meals. The glucose-reducing action of psyllium is proposed due to the formation of a viscous gel in aqueous solution. This gel may hinder the access of glucose to the absorptive epithelium of the small intestine, thereby blunting postprandial glucose peaks. In addition, soluble fiber may delay gastric emptying, thus slowing carbohydrate uptake. Another mechanism that may contribute to the postprandial effect of psyllium is the sequestration of carbohydrates ingested with the meal, thus retarding their access to digestive enzymes (Pal et al., 2014). Also, the hypoglycemic activity of psyllium seeds may be due to the inhibition of liver gluconeogenesis. Furthermore, the increased level of serum insulin by increase insulin secretion from the remnant of $\beta$-cells and enhancement of peripheral metabolism of glucose. Hypoglycemic activity of psyllium seeds may be due to modulating effects on insulin sensitization and/or insulin secretion and a regulating action on digestion and intestinal absorption.

One of the possible mechanism by which psyllium seeds produce hypoglycemic activity is that, Caffeic acid which present at high level in planatago psylium seeds reduce blood glucose level by virtue of their ability to inhibit glucose-6-phosphatase activity, with the related effects on hepatic glucose production and enhancing the glucose kinase (GK) activity in the liver. Hepatic GK has a major effect on glucose homeostasis and is a potential target for pharmacological treatment of Type 2 diabetes. The increase of hepatic GK can cause an increased utilization of the blood glucose for energy production or glycogen storage in the liver (Kalaiarasi and Pugalendi, (2009); and Mohammed et al., 2015).

Moreover, STZ-induced diabetes seems to cause impairment in lipid and lipoprotein metabolism. Also, insulin deficiency is associated with hypercholesterolemia and hypertriglyceridemia due to metabolic abnormalities. In normal conditions, insulin increases the receptor-mediated removal of LDL-C, and decreased activity of insulin during diabetes causes hypercholesterolemia. The increased concentration of TC could result in a relative 
molecular ordering of the residual PL, resulting in a decrease in membrane fluidity (Murali et al., 2002). A good explanation for the increment of TAGs in diabetes is that lipoprotein lipase (LPL), which hydrolyses TAGs into monoglycerides and fatty acids, is inhibited by apolipoprotein CIII and is activated by apolipoprotein CII and insulin. Reduced insulin action may thus increase plasma TAGs by lowering their clearance rate. Enhanced hepatic production of TAGs rich VLDL-C also contributes to the increase in TAGs. Briefly, the transfer of cholesterol ester from HDL-C and LDL-C to triacylglycerols rich lipoprotein (VLDL-C and chylomicrons ), and the transfer of TAGs in the opposite direction is mediated by cholesterol ester transfer protein (CETP), the activity of which is increased in type 2 diabetes TAGs in the LDL-C particle is hydrolyzed by hepatic lipase, making it smaller and denser. In response to elevated concentrations of plasma triacylglycerols-rich lipoproteins, as is typically the case in patients with type 2 diabetes, the rate of transfer is increased. Hyperglycemia is associated with enhanced glycation of LDL-C and an increase in free radical production (Scheffer et al., 2003).

Furthermore, other possible mechanism for hyperlipidemia in DM is explained by Smith and Lall, (2008) that in diabetes, many factors may affect blood lipid levels and this is because carbohydrates and lipid metabolism are interrelated to each other. If there is any disorder in carbohydrate metabolism it also leads to disorder in lipid metabolism. So, there is high concentration of cholesterol, TAGs and due to this there is a reduction in HDL-C levels. Also, insulin deficiency causes excessive metabolism of free fatty acids, this may lead to a disorder in lipid metabolism. Moreover, free fatty acids (FFA) elevation is often associated with hyperlipidemia, which is a risk factor of insulin resistance. Hyperlipidemia includes an increase in TC, FFA, and TAGs levels. These lipid indicators are normally down regulated by insulin. When insulin resistance happens, one or more of these parameters will increase in the blood. Insulin stimulates uptake of FFA by liver and adipose tissue, and conversion of FFA into TAGs. Insulin also induce uptake of TAGs in lipid protein by adipocytes. When adipose tissue lost response to insulin, the levels of FFA and TAGs will increase in the blood.

On the other hand, One of the mechanism of action of psyllium for increasing lipid oxidation and reducing fat may be due to activation of the transient receptor potential vanilloid receptor (TRPV1) channel. It promotes calcium entry that is necessary to prevent preadipocyte-to-adipocyte differentiation. TRPV1 activation may ultimately reduce the number and size of fat cells, and therefore reduce the propensity for obesity to be developed.

Also, another mechanism by which psyllium promotes weight loss may be due to increase the energy expenditure in adipose tissue by stimulating lipolysis and thermogenesis by stimulation of adrenaline secretion from adrenal gland. This increase in serum adrenaline leads to activation of hormone-sensitive lipase (HSL), the key enzyme in the regulation of lipid stores. Moreover, HDL-C protects against or reverse atherosclerosis by their ability to serve as acceptor particles for macrophage cholesterol efflux, prevention of endothelial dysfunction, and maintenance of endothelial integrity (Linsel-Nitschke and Tall, 2005). A decrease in the HDL-C was observed in the present study on diabetic rats, which will increase the chances of atherosclerosis. Treatment with psyllium seeds showed marked elevation in the HDL-C level as compared to that in the controls. The increase in HDL-C is associated with a decrease in coronary risk (Singh et al., 2007).

Moreover, three major hypotheses have frequently been postulated to explain the cholesterol-lowering action of soluble dietary fiber, including psyllium (Anderson et al.,2000; Cornfine et al., 2010; and Anderson et al.,1999). First, the digestion of macronutrients is delayed by viscous soluble fiber, which slows gastric emptying, thus decreasing the transport and mixing of digestive enzymes, and increasing the resistance of the unstirred water layer lining the mucosa to intestinal absorption (Lairon, 2007). This can lead 
to a reduced glycemic response due to a reduction in glucose absorption (Bourdon et al., 1999), and a subsequent decrease in postprandial glucose levels, which is accompanied by a reduction in insulin levels. Decreased insulin levels can then result in the inhibition of 3hydroxy-3-methylglutaryl-coenzyme A reductase (HMG-CoA reductase) and a subsequent reduction in hepatic cholesterol synthesis (Lundin et al.,2004). Second, the presence of soluble fiber in the small intestine may physically prevent bile salt reabsorption into the enterohepatic circulation, resulting in increased fecal bile salt excretion. This may deplete bile salt in the liver, causing cholesterol to be rapidly catabolized in hepatocytes to replenish the bile salt pool via activation of cholesterol 7-alpha-monooxygenase. In addition, there is increased production of LDL-C surface membrane receptors, thus enhancing LDL-C uptake from the bloodstream and consequently lowering cholesterol levels (Marlett et al.,2002; Ellegard and Andersson,2007) .Third, fiber reduces hepatic cholesterol synthesis that is modulated by fermentation products, such as propionate, leading to decreased cholesterol absorption and bile acid reabsorption by physically disrupting the intraluminal formation of micelles (Anderson et al., 2000). It is well known that soluble dietary fibers are not digested or absorbed in the small intestine, but instead undergo anaerobic bacteria fermentation in the cecum and colon to produce short-chain fatty acids (Short-chain fatty acids, particularly propionate, seem to deplete plasma cholesterol by inhibiting hepatic cholesterol metabolism via mechanisms that include reducing $\mathrm{HMG}-\mathrm{CoA}$ receptor activity and inhibiting of acetylCoA reductase, which catalyzes the synthesis of acetyl-CoA from acetate. The hypolipidemic effect of psyllium seeds doesn't seem to be due to only one component ,but rather to the synergetic action of its different constituents, including soluble fiber, phenolic compound, flavonoids ,oelic ,linoleic, linolenic, caffeic acids and chlorogenic acid. Flavonoids may act by making liver cells more efficient to remove LDL-C from blood by increasing LDL-C receptor densities in liver and by binding to apo-lipoprotein B (Gunness et al., 2010)

Moreover, elevated blood sugar in diabetes can trigger direct protein glycation and glucose auto-oxidation which results in molecular and functional changes in proteins and production of free radicals that contribute to development of oxidative stress in STZ-diabetic rats (Ghosh et al., 2015; and Koroglu et al., 2015). Furthermore, increased level of serum glucose, as a reducing agent, can reduce molecular oxygen $\left(\mathrm{O}_{2}\right)$ under physiological conditions, yielding acetaldehydes, hydrogen peroxide $\left(\mathrm{H}_{2} \mathrm{O}_{2}\right)$ and free radical intermediates. Free radical scavenging enzymes such as GPx and G6PD are the first line of defense against injury, and are involved in the disposal of superoxide anions and hydrogen peroxide. The present work have observed decreased activities of GPx and G6PD in diabetic rats and rats fed on HFHC diet, which may be an important factor in limiting the antioxidant capacity because of increased MDA level. Administration of the psyllium seeds along with a HFHC diet caused a significant increase in the activities of these enzymes indicating that the compounds present in the seeds such as flavonoids protect the tissues from lipid peroxidation by their antioxidant ability and consequent reduction in lipid peroxidation. Also, psyllium seeds reversed TAC and MDA changes induced by STZ and HFHC diet. TAC represents a suitable biochemical parameter for evaluating the overall antioxidant status resulting from antioxidant intake or production and their consumption by the increasing level of oxidative stress (Kusan and Ferrari, 2008).

Moreover, the antioxidant activities of psyllium seeds may be due to caffeic acid which present in high levels in psyllium seeds are based on carbonyl group separated from the aromatic ring. The antioxidant mechanism of action is assumed to be through its radicalscavenging activity that is linked to their hydrogen- or electron-donating ability and to the stability of the resulting phenoxyl radicals. It has been documented that caffeic acid possess non-enzymatic antioxidant activity such as scavenging free radicals, and enzymatic 
antioxidant activity such as increasing protein level of antioxidant enzymes (Kalaiarasi and Pugalendi, 2011). In the present study decreasing lipid peroxidation by administration of psyllium seeds may be attributed to psyllium ability to modulate lipid parameters, protein glycation, glucose-autooxidation, thus leading to decrease production of lipid peroxidation products and may be attributed to its antioxidant activity, because psyllium have many phenolic compounds, which have inhibitory effects on lipid peroxidation and preserve the antioxidant compounds

On the other hand, psyllium seeds are rich source of natural antioxidants, PUFAs ( $\omega-3$ and $\omega-6$ fatty acids) and essential and sulfur-rich amino acids, as recommended by the FAO for human health. Psyllium contains phenolics and flavonoids that possess reducing capacity and reactive oxygen species (ROS) scavenging activities. Also, Psyllium contains different metabolites most of the metabolites are natural antioxidants, phenolics, flavonoids, alkaloids and can be used as nutrient supplements. Plantago species are considered to be a natural reservoir of diverse biologically active secondary metabolites, such as lipids, flavonoids, alkaloids, and terpenoids (Patel et al., 2016).

The present work indicates significant increase in hepatic total cholesterol and triacylglycerols in diabetic and hyperlipidemic groups. Ravi et al. (2005) reported that there were a significant increase in the total lipids of liver in STZ-induced diabetic rats and this may indicates an increased synthesis of lipids and storage capacity, which caused an increase in serum triacylglycerols and phospholipids. Authors reported that variety of derangement in metabolic and regulatory mechanisms due to insulin deficiency is responsible for the observed accumulation of lipids. The increase in lipids observed in diabetic rats was due to the impairment of insulin secretion, which resulted in enhanced mobilization of lipid from the adipose tissue to the plasma. Also, Guo et al. (2014) showed that fiber which present in psyllium seeds decreased the concentrations of plasma and liver cholesterol in rats fed with a hypercholesterolemic diet by increasing daily excretions of fecal bile acids.

On the other hand, the histopathological results indicate significant morphological changes in diabetic rats which indicated by decreasing the cellularity of pancreatic $\beta$-cells by replacing it with hyaline deposition. Also, the thickened and hyalinized blood vessels causing not enough oxygen reach the tissue which resulted in degenerative changes. In agreement with our results Erukainure et al. (2013) who showed shrinkage and atrophy of $\beta$-cells of islets of Langerhans in the diabetic animals compared to normal islets in control group. Then treatment with fiber -enriched cake increased the number of islets as compared to diabetic untreated animals.

Our results indicated the role of psyllium in mediating mechanisms in protecting the pancreatic tissue from diabetes induced disorders. The reduction of hyperglycemia may be a cause of such protection. Our study provides valuable information that supports the possibility of preventing or at least attenuating pancreatic island damage in diabetes by diet supplementation with psyllium which contain high amount of antioxidants.

\section{Conclusion:}

The results of the present study showed that diet supplemented with psyllium seeds showed hypoglycemic hypolipidemic effects in addition of having significant antioxidant prosperities. Therefore, it was concluded that plantago psyllium seeds may be considered helpful to lower glucose and lipid levels in treatment of hyperglycemia and hyperlipidemia as well as protect against cardiovascular diseases risk. 


\section{References:}

1- Ahmed I. , Naeem M., Shakoor A., Ahmed Z., and Iqbal H (2010) : Investigation of Anti-diabetic and Hypocholesterolemic Potential of Psyllium Husk Fiber (Plantago psyllium) in Diabetic and Hypercholesterolemic Albino Rats. Int.J.Bio.lif.sci. ; 6 (3) :185-189

2- American Diabetes Association (2014): Diagnosis and classification of diabetes mellitus. Diabetes Care; 37(1): 81-90.

3- Anderson J.W., Allgood L.D., Turner C., Oelgten P.R., and Daggy B.P. (1999): Effects of psyllium on glucose and serum lipid responses in men with type 2 diabetes and hypercholesterolemia. Am.J.Clin.Nutr.;70:466-473.

4- - Anderson J.W., Hanna T.J., Peng X., and Kryscio R.J. ( 2000):Whole grain foods and heart disease risk. J. Am. Coll. Nutr.;19: 291-299.

5- Aune D., Norat T., Romundstad P., and Vatten L.J. (2013): Whole grain and refinedgrain consumption and the risk of type 2diabetes: a systematic review and dose-response meta-analysis of cohort studies. Eur .J .Epidemiol; 28:845-858.

6- Bhateja P.K., and Singh R. (2016): Antioxidant activity of acacia tortilis polysaccharide in streptozotocin-nicotinamide induced diabetic rats. Int. J. Toxicol. Pharmacol. Res. ;8 (3): 152-162

7- Bourdon I., Yokoyama W., Davis P., Hudson C., Backus R., Richter D., Knuckles B., and Schneeman B.O. (1999): Postprandial lipid, glucose, insulin and cholrcystokinin responses in men fed barley pasta enriched with beta-glucan. Am. J. Clin. Nutr. ; 69: 55-63.

8- Brehm A., Pfeiler G., Pacini G., Vierhapper H., and Roden , M. (2004): Relationship between serum lipoprotein ratios and insulin resistance in obesity. Clin.Chem. ; 50: 2316-2322.

9- Cornfine C., Hasenkopf K., Eisner P., and Schweiggert U. (2010): Influence of chemical and physical modification on the bile acid binding capacity of dietary fiber from lupins (Lupinusangustifolius L.). Food Chem. ; 122 (3): 638-644.

10-Dror Y., Sassoon H.F., Watson J.J. and Johnson B.C (1970): Glucose-6phosphate dehydrogenase assay in liver and blood. Clin. Chem. Acta.; 28: 291298.

11- Drury R.A.B. and Wallington E.A.(1980): Preparation and fixation of tissues. In: Drury RAB, Wallington EA, editors. Carleton's Histological Technique. 5. Oxford; 41-54. 
12- Ellegard L., and Andersson H. (2007): Oat bran rapidly increases bile acid excretion and bile acid synthesis: an ileostomy study.Eur.J.Clin.Nut.;61 : 938-945.

13- Erukainure O.L., Ebuehi O.A.T., Adeboyejo F.O., Aliyu M., and Elemo G.N.(2013): Hematological and biochemical changes in diabetic rats fed with fiber-enriched cake. J. A. M . ; 3(2) : 39-44.

14- Folch J., Lees M., and Sloane S.G.H (1957): A simple method for isolation and purification of total lipids from animal tissues. J .Biol. Chem.; 26:497-509.

15- Fossati P., and Prencipe L.(1982): Serum triglycerides determined colorimetrically with an enzyme that produces hydrogen peroxide. Clin.Chem; 28:2077-2080.

16- Fridewald W.T., Levei R.I. and Fridrickson D.S. (1972) :Estimation of the concentration of low density lipoproteins cholesterol in plasma without use of the ultra centrifugation. Clin. Chem. Res. ; $18: 499-502$.

17- Garg N., Agrawal. Y. B., and Gupta. S. (2014) :A study of lipid profile levels in diabetics and non-diabetics taking $\mathrm{TC} / \mathrm{HDL}$ ratio and $\mathrm{LDL} / \mathrm{HDL}$ ratio into consideration. J.I. A. C. M; 15(3-4): 192-195.

18- Ghosh S., Banerjee S., and Sil P.C. (2015): The beneficial role of curcumin on inflammation, diabetes and neurodegenerative disease: A recent update. Food Chem. Toxicol. ;83:111-124.

19- Guang-Kai .X.U. , Xiao-Ying Q.I.N., Guo-Kai W.A.N.G, Guo-Yong. X.I.E, Xu-Sen .L.I., Chen-Yu S.U.N., Bao-Lin L.I.U. and Min-Jian Q.I.N (2017): Antihyperglycemic, antihyperlipidemic and antioxidant effects of standard ethanol extract of Bombaxceiba leaves in high-fat-diet- and streptozotocin-induced Type 2 diabetic rats. Chin. J. Natu. Med; 15(3): 168- 177.

20- Gunness P ., and Gidley M.J. (2010): Mechanisms underlying the cholesterollowering properties of soluble dietary fiber polysaccharides. Food Funct.;1(2):149155.

21- Guo, C.H., Huang, C. J., Chiou, X. I., and Hsu, G.S.W. (2002): Alteration of trace element distribution and testis ACE activity in mice with high peritoneal aluminum. Bio. Tra. Elem. ; 85:145-157.

22- Guo L., Tong L.T., Liu L., Zhong K., Qiu J.U., and Zhou S. (2014):The cholesterol-lowering effects of oat varieties based on their difference in the composition of proteins and lipids. Lipids in Health and Disease; 13: 1-182.

23- Haddadian K, Haddadian K, and Zahmatkash M. A (2014):A review of plantago plant. Ind. J. Tra. Knowl. ; 13 (4) :681-685.

24- Haroun M.A., Elsayed .L.A., Rashed L.A. and Mohamed M .A. (2011): The effect of high fat diet and high fructose intake on insulin resistance and GLP-1 in experimental animals. Med. J. Cairo Univ.; 79 (2) : 23-32 
25- Johnson K.R., Johnston C.S., and Sweazea K.L. (2017) :Ground flaxseed increased nitric oxide levels in adults with type 2 diabetes: A randomized comparative effectiveness study of supplemental flaxseed and psyllium fiber. Obes. Med.; 5: 16-24.

26- Kalaiarasi p., and Pugalendi K.V. (2009): Antihyperglycemic effect of $18 \beta$ glycyrrhetinic acid, aglycone of glycyrrhizin, on streptozotocin-diabetic rats. Europ. J. Pharmacol.; 606(1-3) 269-273.

27- Kalaiarasi p., and Pugalendi K.V. (2011): Protective effect of 18b-glycyrrhetinic acid on lipid peroxidation and antioxidant enzymes in experimental diabetes. J.Ph. Res. ;4 (1) : 107- 111.

28- Kaplan A. (1984): Quantitative determination of total lipids. Clin. Chem; 22: 919932.

29- Karhunen L.J., Juvonen K.R., and Flander S.M (2010): A psyllium fiberenriched meal strongly attenuates postprandial gastrointestinal peptide release in healthy young adults. J. Nutr. ;40:737-744.

30- Koracevic D., Koracevic G., Djordjevic V., Andrejevic S., and Cosic V.(2001): Colorimetric method for determination of total antioxidant capacity. Pathology; 54 :356-361.

31- Koroglu P., Senturk G.E., Yucel D., Ozakpinar O.B., Uras F., and Arbak S. (2015): The effect of exogenous oxytocin on streptozotocin (STZ)-induced diabetic adult rat testes. Peptides; $63: 47-54$.

32- Kusan C., and Ferrari B. (2008): Total antioxidant capacity a biomarker in biomedical and nutritional studies. J. Cell Mol. Biol;7:1-15.

33- Lairon D. (2007): Dietary fiber and control of body weight. Nutrition, metabolism, and cardiovascular diseases: N.M.C.D; 17 (1): 1-5.

34- Latha S., Rajaram K., and Kumar p.s. (2014): Hepatoprotective and antidiabetic effect of methanol extract of caralluma fimbriata in STZ induced diabetic albino rats. Int. J. Phar. Pharmace. Sci ; 6 (1) : 975-1491

35- Lecumberri E., Goya L., Mateos R., Alía M., Ramos S., Izquierdo-Pulido M., and Laura B. (2007): A diet rich in dietary fiber from cocoa improves lipid profile and reduces malondialdehyde in hypercholesterolemic rats. J. Nut ; 23 : 332-341.

36- Levesque R. (2007): SPSS Programming and Data Management: A Guide for SPSS and SAS users. 3rd Edition. SPSS Inc, United States of America. 
37- Linsel-Nitschke P.I., and Tall A.R.(2005): HDL as a target in the treatment of atherosclerotic cardiovascular disease. Nat. Rev. Drug Discov.; 4:193-205.

38- Lopez-Virella M.F., Stone P., Ellis S. and Colwell J.A (1977): Cholesterol determination in high-density lipoproteins separated by three different methods. Clin. Chem. ;23 (5) 882-884.

39- Lundin E., Zhang J.X., Hallmans G., Lairon D., Tidehag P., Åman P., and Adlercreutz H. (2004): Effects of meal frequency and high-fiber rye-bread diet on glucose and lipid metabolism and ileal excretion of energy and sterols in ileostomy subjects. Eur. J.Clin.Nut ;58:1410-1419.

40- Marlett J.A., McBurney M.I., and Slavin J.L. (2002): American dietetic association: health implications of dietary fiber. J. Am. Diet. Assoc.;102: 9931000 .

41- Matthews D.R., Hosker J.P., Rudenski A.S., Naylor B.A., Treacher D.F. andTurner .R.C.(1985): Homeostasis model assessment: insulin resistance and beta-cell function from fasting plasma glucose and insulin concentrations in man. J.Diabetologia.; 28(7):412-419

42-Mohamed F. Z., AL-hussini A.S. and EL- shehabi M.E. (2015): Anti-diabetic activity of caffeic acid and $18 \beta$ glycyreetinic acid and its relationship with the antioxidants properties.Asian.J.Pharm.Clin.Res.;8( 5) :229-234.

43- Murali B., Upadhyaya U.M, and Goyal R.K (2002): Effect of chronic treatment with Enicosistemmalittorale in non-insulin-dependent diabetic (NIDDM) rats. J. Ethnopharmacol.;81:199-204.

44- Notarnicola M., Altomare D.F., and Correale .M. (2005): Serum lipid profile in colorectal cancer patients with and without synchronous distant metastases. Onc.;68(4-6):371-374.

45- Ohkawa H., Ohishi N., and Yagi K (1979): Assay for lipid peroxides in animal tissues by thiobarbituric acid reaction. Anal. Biochem.; 95: 351-358.

46- Paglia D. E. and Valentina W.N. (1967): Studies on the quantitative and qunlitative characterization of erytrocyte glutathione peroxidase. J.Lab.Clin Med.; 70: 158-169.

47- Pal S., Radavelli-Bagatini S., Ho S., McKay J. and Jane M. (2014): Using psyllium to prevent and treat obesity comorbidities. In nutrition in the prevention and treatment of abdominal obesity. Elsevier Inc.: 505-514.

48- Patel M. K., Mishra A., and Jha B. (2016): Non-targeted metabolite profiling and scavenging activity unveil the nutraceutical potential of psyllium (Plantago ovataForsk). Frontiers in Plant Sci. ;7: 431. 
49- Pengzhan Y., Quanbin Z., Ning L., Zuhong X., Yanmei W. and Zhien L. (2003): Polysaccharides from Ulvapertusa (Chlorophyta) and preliminary studies on their antihyperlipidemia activity. J. Appl. Phycol.;15(1):21-27.

50- Rajagopal K., and Sasikala K.(2008): Antidiabetic activity of hydro-ethanolic extracts of NymphaeaStellataflowers in normal and alloxan induced diabetic rats. Afr. J. Pharma. Pharmacol.; 2(8) : 173-178.

51-Ravi K., Rajasekaran S., and Subramanian S. (2005): Antihyperlipidemic effect of Eugenia jambolana seed kernel on streptozotocin-induced diabetes in rats. Food .Chem. Toxic. ;43: 1433-1439.

52- Reeves P. G., Nielsen F. H. and Fahey G. C. (1993) :AIN-93 purified diets for laboratory rodents: final report of the American Institute of Nutrition ad hoc writing committee on the reformulation of the AIN-76A rodent diet. J. Nutr. ;123: 1939-1951.

53- Ribeiro D., Pinto-Fochi M.E., Rafacho A., and Goes .R.(2012) : High-Fat Diet Obesity Associated with insulin resistance increases cell proliferation, estrogen receptor, and PI3K proteins in rat ventral prostate. J.Andro.; 33 (5) : 854-865.

54- Richmond W.(1973):Preparation and prosperities of a cholesterol oxidase from Norcadia sp. and its application to the enzymatic assay of total cholesterol in serum.Clin.Chem.;19:1350-1356.

55-Satija A., and Hu F.B. (2012): Cardiovascular benefits of dietary fiber. Current Atherosclerosis Reports; 14: 505-514.

56- Scheffer P.G., Bos G., Volwater H.G., Dekker J.M., Heine R.J.,andTeerlink T. (2003):.Associations of LDL size with in vitro oxidizability and plasma levels of in vivo oxidized LDL in Type 2 diabetic patients. Diabet. Med.; 20(7):563-567.

57-Singh I.M., Shishehbor M.H., and Ansell B.J. (2007): High-density lipoprotein as a therapeutic target: a systematic review J.A.M.A; 298: 786-798.

58- -Smith S. and Lall A.M (2008): A Study on lipid profile levels of diabetics and non-diabetics among Naini region of Allahabad, India. Turk.J. Biochem.; 33(4):138-141.

59- Spin R., Ongagna J.C., Gasser F. and Grucker D.(1998):Insulin measurements in haemolysed serum; influence of insulinas inhibitors. Clin. Chim. Acta ; 274:111-117.

60-Suresh D.R, Annam .V, and Krishnappa P.H. (2010): Immunological correlation of oxidative stress markers in tuberculosis patients. Int.J.Biol.Med.Res.;1(4):185-7. 
61- Tietz N.W. (1990): Clinical Guide to Laboratory Test. 2nd Edn, W.B. Saunders company, Philadelphia, U.S.A: 554-556.

62- Trinder P. (1969): Determination of glucose in blood using glucose oxidase with an alternative oxygen acceptor. Ann. Clin. Biochem.; 6: 158-161.

63- Uttra K.M., Devrajani B.R., Shah Z.A., Devrajani T., Das T., and Raza S. (2011): Lipid profile of patients with diabetes mellitus (A Multidisciplinary Study). World Appl. Sci J; 12(9):1382-1384.

64- Van Rosendaal G.M., Shaffer E.A., Alun L.E, and Brant R (2004): Effect of administration on cholesterol-lowering by psyllium: a randomized cross-over study in normocholesterolemic or slightly hypercholesterolemic subjects. J. Nutr; 28: 317.

65- Wilson R.D and Islam M.S.(2012):Fructose-fed streptozotocin-injected rat: an alternative model for type 2 diabetes. Pharmacol Rep.; 64(1):129-39. 
"تأثير تناول بذور السيليوم علي خفض سكر ودهون الام في الجرذان المصابه بالسكري وارتفاع دهون الدم "

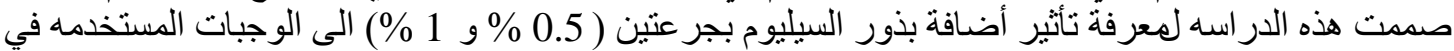

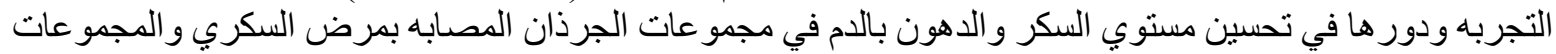

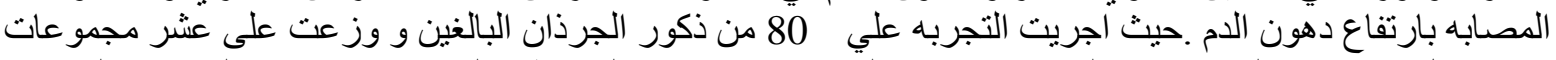

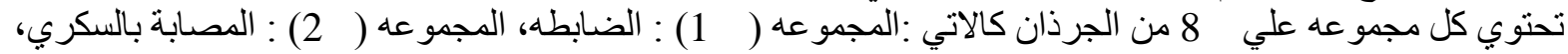

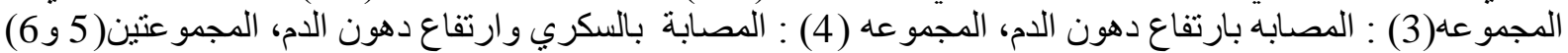

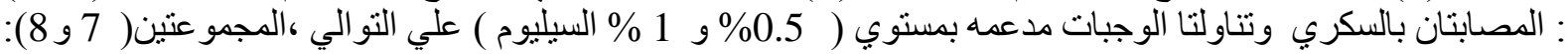

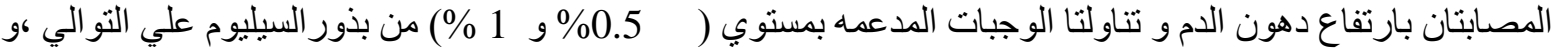

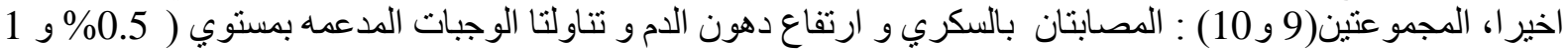

واستمرت التجربة لمدة 6 اسابيع ثم تم ذبح الجرذان بعد صوم 12 ساعه و وتم تجميع عينات الدم و الاعضاء (الكبد \% \% السيليوم).

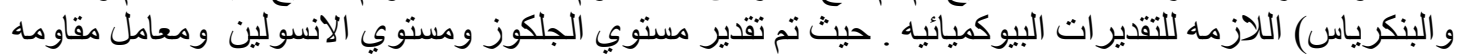

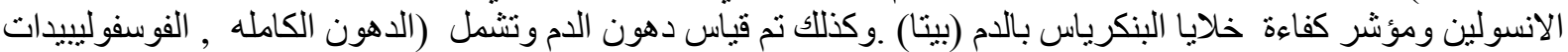

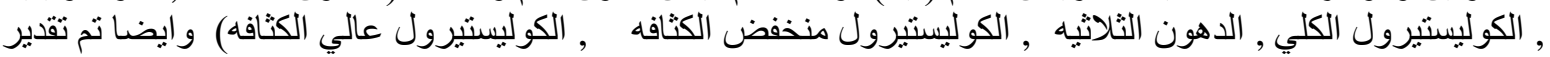

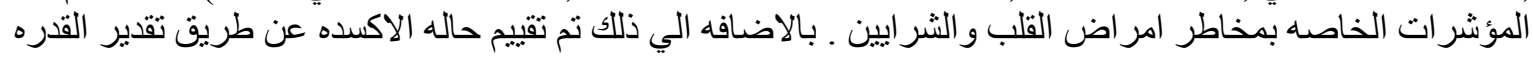

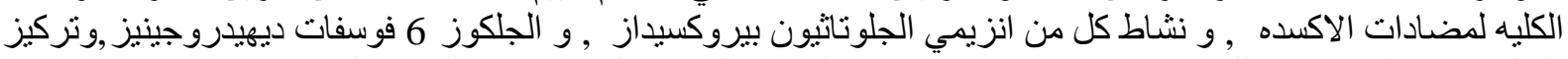

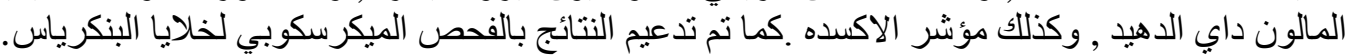

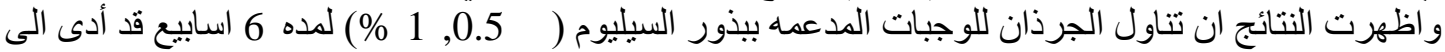

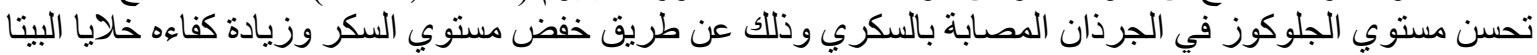

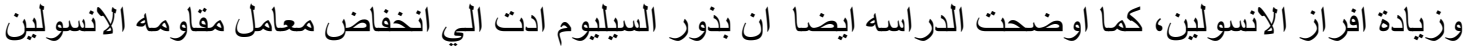

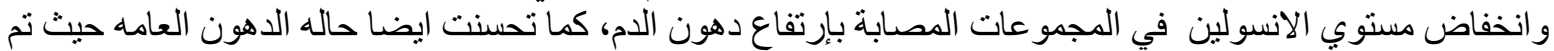

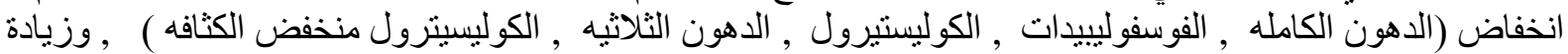

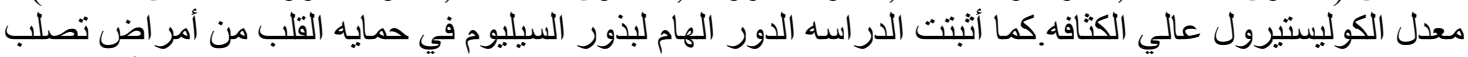

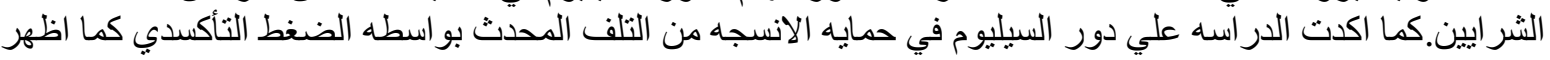

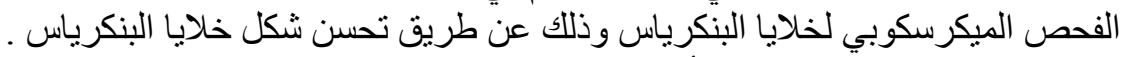

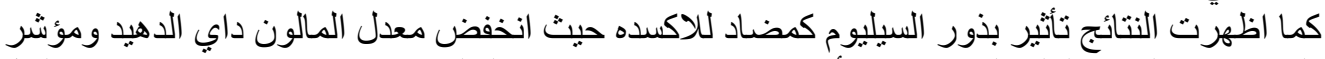

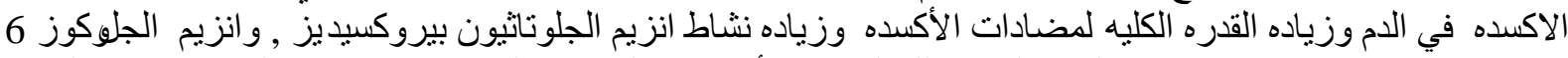

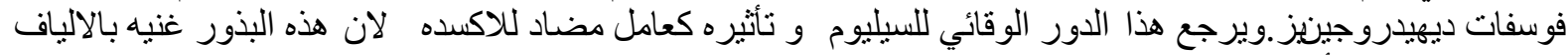
و الفلافونيدات و الأحماض الدهنية غير المشبعة التي بدور ها تحمي الخلايا من الثنقوق الحرها

$$
\text { ا.د/زكيه مصطفي عبدالقادر }
$$

أستاذعلوم الاغذيه بقسم الكيمياء الحيويةو التغذية كلية البناتـ جامعه عين شمس

$$
\text { دكتورة /هناء مصطفي عبد الفتاح }
$$

أستاذ التخذيه بقسم الكيمياء الحيوية و التغذية كلية البنات- جامعه عين شمس

$$
\text { دكتوره/مي السيد }
$$

محاضر بقسم الكمياء الحيو يه والتغذيه بكليه البنات -جامعه عين شمس

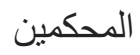

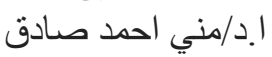

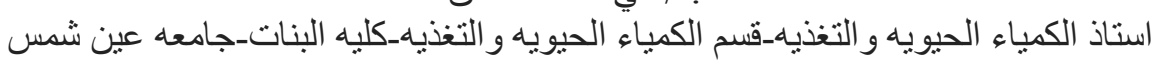

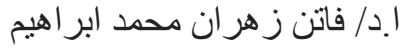

$$
\begin{aligned}
& \text { استاذ الكمياء الحيويه ـاستاذ الكمباء الحيويه_كليه العلوم -جامعه الزقازيق النيق }
\end{aligned}
$$


